\title{
Application Crash Consistency and Performance with CCFS
}

\author{
THANUMALAYAN SANKARANARAYANA PILLAI, RAMNATTHAN ALAGAPPAN, and \\ LANYUE LU, University of Wisconsin - Madison \\ VIJAY CHIDAMBARAM, The University of Texas at Austin \\ ANDREA C. ARPACI-DUSSEAU and REMZI H. ARPACI-DUSSEAU, \\ University of Wisconsin - Madison
}

\begin{abstract}
Recent research has shown that applications often incorrectly implement crash consistency. We present the Crash-Consistent File System (ccfs), a file system that improves the correctness of application-level crash consistency protocols while maintaining high performance. A key idea in ccfs is the abstraction of a stream. Within a stream, updates are committed in program order, improving correctness; across streams, there are no ordering restrictions, enabling scheduling flexibility and high performance. We empirically demonstrate that applications running atop ccfs achieve high levels of crash consistency. Further, we show that ccfs performance under standard file-system benchmarks is excellent, in the worst case on par with the highest performing modes of Linux ext4, and in some cases notably better. Overall, we demonstrate that both application correctness and high performance can be realized in a modern file system.
\end{abstract}

CCS Concepts: • General and reference $\rightarrow$ Reliability; Performance; $・$ Information systems $\rightarrow$ File systems; • Software and its engineering $\rightarrow$ File systems management;

Additional Key Words and Phrases: File systems, crash consistency, reordering, performance

\section{ACM Reference format:}

Thanumalayan Sankaranarayana Pillai, Ramnatthan Alagappan, Lanyue Lu, Vijay Chidambaram, Andrea C. Arpaci-Dusseau, and Remzi H. Arpaci-Dusseau. 2017. Application Crash Consistency and Performance with CCFS. ACM Trans. Storage 13, 3, Article 19 (September 2017), 29 pages.

https://doi.org/10.1145/3119897

\footnotetext{
The research herein was supported by funding from NSF grants CNS-1419199, CNS-1421033, CNS-1319405, and CNS-1218405, DOE grant DE-SC0014935, as well as donations from EMC, Facebook, Google, Huawei, Microsoft, NetApp, Samsung, Seagate, Veritas, and VMware. Any opinions, findings, and conclusions or recommendations expressed in this material are those of the authors and may not reflect the views of NSF, DOE, or other institutions. This article is an extended version of a FAST '17 paper by T.S. Pillai et al. [37]. The additional material here includes a detailed description of the stream API, detailed discussion of the limitations of the stream abstraction and the ccfs file system, more thorough discussion of developer effort required to make applications crash consistent atop ccfs, more figures and code snippets to illustrate usage of streams, and several other minor edits and updates.

Authors' addresses: T. S. Pillai, R. Alagappan, and L. Lu, 1210 W. Dayton St., Madison, WI 53706; V. Chidambaram, 2317 Speedway, Austin, TX 78712; A. C. Arpaci-Dusseau and R. H. Arpaci-Dusseau, 1210 W. Dayton St., Madison, WI 53706; emails: \{madthanu, ra, 1l\}@cs.wisc.edu, vijay@cs.utexas.edu, \{dusseau, remzi\}@cs.wisc.edu.

Permission to make digital or hard copies of all or part of this work for personal or classroom use is granted without fee provided that copies are not made or distributed for profit or commercial advantage and that copies bear this notice and the full citation on the first page. Copyrights for components of this work owned by others than the author(s) must be honored. Abstracting with credit is permitted. To copy otherwise, or republish, to post on servers or to redistribute to lists, requires prior specific permission and/or a fee. Request permissions from permissions@acm.org.

2017 Copyright is held by the owner/author(s). Publication rights licensed to ACM.

ACM 1553-3077/2017/09-ART19 \$15.00
}

https://doi.org/10.1145/3119897

ACM Transactions on Storage, Vol. 13, No. 3, Article 19. Publication date: September 2017. 


\section{INTRODUCTION}

"Filesystem people should aim to make 'badly written' code 'just work."” -Linus Torvalds [56]

The constraint of ordering is a common technique applied throughout all levels of computer systems to ease the construction of correct programs. For example, locks and condition variables limit how multi-threaded programs run, making concurrent programming simpler [3]; memory consistency models with stricter constraints (e.g., sequential consistency) generally make reasoning about program behavior easier [51]; fsync calls in data management applications ensure preceding I/O operations complete before later operations [6, 38].

Unfortunately, constraining ordering imposes a fundamental cost: poor performance. Adding synchronization primitives to concurrent programs adds overhead and reduces performance [20, 22]; stronger multiprocessor memory models are known to yield lower throughput [17]; forcing writes to a disk or SSD can radically reduce I/O performance $[6,7]$. While in rare cases we can achieve both correctness and performance [43], in most cases we must make an unsavory choice to sacrifice one.

Within modern storage systems, this same tension arises. A file system, for example, could commit all updates in order, adding constraints to ease the construction of applications (and their crash-recovery protocols) atop them $[4,38]$. Many file system developers have determined that such ordering is performance prohibitive; as a result, most modern file systems reduce internal ordering constraints. For example, many file systems (including ext4, xfs, btrfs, and the 4.4BSD fast file system) re-order application writes [2], and some file systems commit directory operations out of order (e.g., btrfs [38]). Lower levels of the storage stack also re-order aggressively, to reduce seeks and obtain grouping benefits [23, 24, 45, 47].

However, research has shown that user-level applications are often incorrect because of reordering $[1,38,60]$. Many applications use a specialized write protocol to maintain crash consistency of their persistent data structures. The protocols, by design or accident, frequently require all file-system updates to commit in their issued order [39].

The main hypothesis in this article is that a carefully designed and implemented file system can achieve both ordering and high performance. We explore this hypothesis in the context of the Crash-Consistent File System (ccfs), a new file system that enables crash-consistent applications while delivering excellent performance.

The key new abstraction provided by ccfs, which enables the goals of high performance and correctness to be met simultaneously, is the stream. Each application's file-system updates are logically grouped into a stream; updates within a stream, including file data writes, are guaranteed to commit to disk in order. Streams thus enable an application to ensure that commits are ordered (making recovery simple); separating updates between streams prevents false write dependencies and enables the file system to re-order sufficiently for performance.

Underneath this abstraction, ccfs contains numerous mechanisms for high performance. Critically, while ordering updates would seem to overly restrict file-system implementations, we show that the journaling machinery found in many modern systems can be adapted to yield high performance while maintaining order. More specifically, ccfs uses a novel hybrid-granularity journaling approach that separately preserves the order of each stream; hybrid granularity further enables other needed optimizations, including delta journaling and pointer-less metadata structures. Ccfs takes enough care to retain optimizations in modern file systems (like ext4) that appear at first to be incompatible with strict ordering, with new techniques such as order-preserving delayed allocation. 


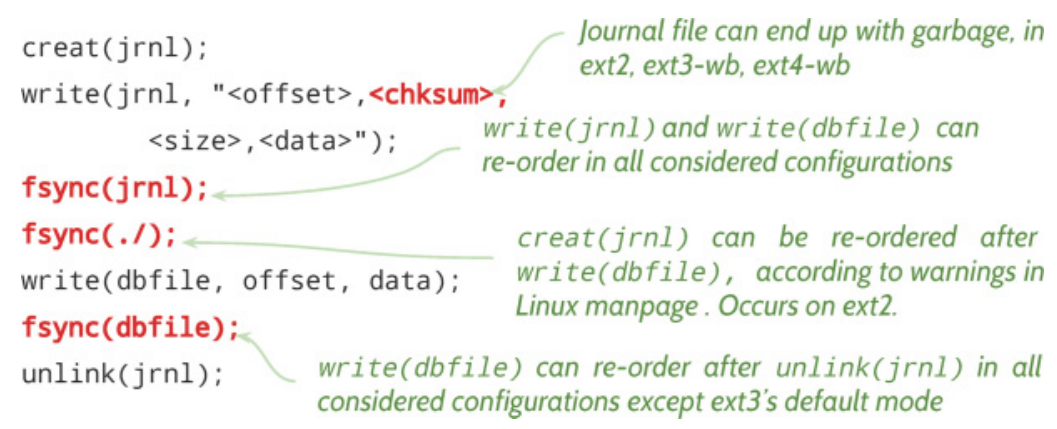

Fig. 1. Journaling Update Protocol. Pseudo-code for a simple version of write-ahead journaling; each statement is a system call. The normal text corresponds directly to the protocol's logic, while the bold parts are additional measures needed for portability. Italicized comments show which measures are needed under the default modes of ext2, ext3, ext4, xfs, and btrfs, and the writeback mode of ext3/4 (ext3-wb, ext4-wb).

We show that the ordering maintained by ccfs improves correctness by testing five widely used applications (Git, LevelDB, ZooKeeper, Mercurial, and SQLite), four of which (all except SQLite) are inconsistent on ext4 and other modern file systems [38]. We also show that the performance of standard benchmarks on ccfs are on par with ext4. We compare the performance of three real-world applications (Git, LevelDB, and SQLite) on ccfs and ext4; SQLite and LevelDB perform similarly on both file systems and Git has a $80 \times$ performance improvement on ccfs, when modified on ext4 to achieve the same correctness as on ccfs. Furthermore, with additional developer effort, the performance of SQLite and LevelDB on ccfs can be improved by factors of $685 \times$ and $5 \times$, respectively. We analyze in detail the developer effort required for applications to use the stream abstraction and conclude it is trivial; for instance, we added a single line of code to Git to achieve both crash consistency and efficiency atop ccfs, while many complicated changes to many source files are required to make Git crash-consistent atop re-ordering file systems.

The article is structured as follows. We provide motivation and background (Section 2), present ccfs (Section 3), and evaluate it (Section 4). We then discuss the limitations of streams and ccfs (Section 5). Finally, we discuss related work (Section 6) and conclude (Section 7).

\section{MOTIVATION AND BACKGROUND}

In this section, we first explain the extent to which current data-intensive applications are vulnerable during a crash. We then describe why a file system that preserves the order of application updates will automatically improve the state of application-level crash consistency. Finally, we discuss the performance overheads of preserving order and how the overheads can be addressed.

\subsection{State of Crash Consistency}

To maintain the consistency of their user-level data structures in the event of a crash, many applications $[21,26,34]$ modify the data they store in the file system via a carefully implemented update protocol. The update protocol is a sequence of system calls (such as file writes and renames) that updates underlying files and directories in a recoverable way. As an example, consider a simple DBMS that stores its user data in a single database file. To maintain transactional atomicity across a system crash, the DBMS can use an update protocol called journaling (or write-ahead logging): before updating the database file, the DBMS records the updates in a separate journal file. The pseudocode for the update protocol is shown in Figure 1. If a crash happens, then the 
DBMS executes a recovery protocol when restarted: if the database file was only partially updated, the full update from the journal is replayed.

Correctly implementing crash-consistency protocols has proven to be difficult for a variety of reasons. First, their correctness inherently depends on the exact semantics of the system calls in the update protocol with respect to a system crash. Because file systems buffer writes in memory and send them to disk later, from the perspective of an application the effects of system calls can get re-ordered before they are persisted on disk. For example, in a naive version of the journaling update protocol, the unlink of the journal file can be re-ordered before the update of the database file. In Figure 1, an explicit fsync system call is used to force the update to disk before issuing the unlink. Also, the semantics of system calls can differ between file systems; for example, the aforementioned re-ordering occurs in the default configurations of ext2, ext4, xfs, and btrfs, but not in ext3.

Second, the recovery protocol must correctly consider and recover from the multitude of states that are possible when a crash happens during the update protocol. Application developers strive for update protocols to be efficient, since the protocols are invoked during each modification to the data store; more efficient update protocols often result in more possible states to be reasoned about during recovery. For example, the journal protocol in Figure 1 is often extended to batch multiple transactions onto the journal before the actual update to the database file, to avoid performanceintensive fsync calls.

Finally, crash-consistency protocols are hard to test, much like concurrency mechanisms, because the states that might occur on a crash are non-deterministic. Since efficient protocol implementations are inherently tied to the format used by the application's data structures and concurrency mechanisms, it is impractical to re-use a single, verified implementation across applications.

Unsurprisingly, past research $[38,60,61]$ has found many vulnerabilities in the implementations of crash consistency protocols in widely used applications written by experienced developers, such as Google's LevelDB and Linus Torvalds's Git. However, in this article, we argue that it is practical to construct a file system that automatically improves application crash consistency. We base our arguments on the following hypotheses:

The Ordering Hypothesis: Existing update and recovery protocols (mostly) work correctly on an ordered and weakly atomic file system (we define these terms subsequently).

The Efficiency Hypothesis: An ordered and weakly atomic file system can be as efficient as a file system that does not provide these properties, with the proper design, implementation, and realistic application workloads.

\subsection{Weak Atomicity and Order}

We hypothesize that most vulnerabilities that exist in application-level update protocols are caused because the related application code depends on two specific file-system guarantees. File systems that provide these guarantees, therefore, automatically mask application vulnerabilities. The first guarantee, and the major focus of our work, is that the effect of system calls should be persisted on disk in the order they were issued by applications; a system crash should not produce a state where the system calls appear re-ordered. The second (minor) guarantee is that, when an application issues certain types of system calls, the effect of the system call should be atomic across a system crash. The second guarantee, which we term weak atomicity, is specifically required for system calls that perform directory operations (including the creation, deletion, and renaming of files and hard links). Weak atomicity also includes stipulations about writes to files, but only at sector granularity (i.e., there is generally no need to guarantee that arbitrarily large writes are atomic). If a system call appends data to the end of a file, then both increasing the file size and the writing of data to the newly appended portion of the file should be atomic together. 
Table 1. Seeks and Order

\begin{tabular}{c|ccc}
\hline & Time (s) & Seeks & Median seek distance (sectors) \\
\hline Re-ordered & 25.82 & 23,762 & 120 \\
FIFO & 192.56 & 38,201 & 2002112 \\
\hline
\end{tabular}

The table shows the number of disk seeks incurred and the total time taken when 25,600 writes are issued to random positions within a 2GB file on a Toshiba MK1665GSX 160GB HDD (our experimental setup is described in more detail in Section 4). Two different settings are investigated: the writes can be re-ordered or the order of writes is maintained using the FIFO strategy. The number of seeks incurred in each setting and the LBA seek distance shown are determined from a block-level I/O trace.

The fundamental reason that order simplifies the creation of update protocols is that it drastically reduces the number of possible states that can arise in the event of a crash, that is, the number of states that the recovery protocol has to handle. For example, consider an update protocol that simply overwrites $n$ sectors in a file; if the file system maintains order and weak atomicity, only $n$ crash states are possible, whereas $2^{n}$ states are possible if the file system can re-order. Maintaining order makes it easier to reason about the correctness of recovery for both humans and automated tools [38].

The effectiveness of maintaining weak atomicity and order can be understood by considering the application-level crash vulnerabilities discovered in our previous study [38]. Among 60 vulnerabilities, we found that 16 are masked by maintaining weak atomicity alone. We also found that 27 vulnerabilities are masked by guaranteeing order. Of the remaining vulnerabilities, 12 are attributed to durability; however, we observe that 8 of these 12 will be masked if the file system guarantees order. Thus, in all, 50 of the 60 vulnerabilities are addressed by maintaining order and weak atomicity; the remaining 10 have minor consequences and are readily masked or fixed [39].

\subsection{Order: Bad for Performance}

Most real-world deployed file systems (such as btrfs) already maintain the weak atomicity required to mask application-level crash-consistency vulnerabilities. However, all commonly deployed filesystem configurations (including ext4 in metadata-journaling mode, btrfs, and xfs) re-order updates, and the re-ordering only seems to increase with each new version of a file system (e.g., ext4 re-orders more than ext3 [38]; newer versions of ext4 re-order even more [57], as do newer systems like btrfs [38]). While maintaining update order is important for application crash consistency, it has traditionally been considered bad for performance, as we now discuss.

At low levels in the storage stack, re-ordering is a fundamental technique that improves performance. To make this case concrete, we created a workload that issues writes to random locations over a disk. Forcing these writes to commit in issue order takes roughly eight times longer than a seek-optimized order (Table 1). Re-ordering is important for hard drives [47] and SSDs [24]; approaches that constrict write ordering are insufficient.

Higher up the stack, ordering can induce negative (and sometimes surprising) performance degradations. Consider the following code sequence:

$$
\text { write }\left(f_{1}\right) \text {; write }\left(f_{2}\right) \text {; fsync }\left(f_{2}\right) \text {; truncate }\left(f_{1}\right) \text {; }
$$

In this code, without mandated order, the forced writes to $f_{2}$ can move ahead of the writes to $f_{1}$; by doing so, the truncate obviates the need for any writes to $f_{1}$ at all. Similarly, if the user overwrites $f_{1}$ instead of truncating it, only the newer data needs to be written to disk.

We call this effect write avoidance: not all user-level writes need to be sent to the disk, but can instead be either forgotten due to future truncates or coalesced due to future overwrites. Reordering allows write avoidance across fsync calls. Global write ordering, in contrast, implies that 
if writes to $f_{2}$ are being forced to disk, so must writes to $f_{1}$. Instead of skipping the writes to $f_{1}$, the file system must now both write out its contents (and related metadata), and then, just moments later, free said blocks. If the write to $f_{1}$ is large, then this cost can be high.

We call this situation, where fsync calls or cache eviction reduce write avoidance in an ordered file system, a write dependence. Write dependence is not limited to writes by a single application; any application that forces writes to disk could cause large amounts of other (potentially unneeded) I/O to occur. When write dependence does not improve crash consistency, as when it occurs between independent applications, we term it a false dependence, an expected high-cost of global order.

Apart from removing the chance for write avoidance, write dependence also worsens application performance in surprising ways. For example, the $\operatorname{fsync}\left(f_{2}\right)$ becomes a high-latency operation, as it must wait for all previous writes to commit, not just the writes to $f_{2}$. The overheads associated with write dependence can be further exacerbated by various optimizations found in modern file systems. For example, the ext4 file system uses a technique known as delayed allocation, wherein it batches together multiple file writes and then subsequently allocates blocks to files. This important optimization is defeated by forced write ordering.

\subsection{Order with Good Performance}

We believe it is possible to address the overheads associated with maintaining order in practice. To reduce disk-level scheduling overheads, a variety of techniques have been developed that preserve the appearance of ordered updates in the event of a crash while forcing few constraints on disk scheduling.

For example, in ext4 data journaling, all file-system updates (metadata and data) are first written to a journal. Once committed there, the writes can be propagated (checkpointed) to their in-place final locations. Note that there are no ordering constraints placed upon the checkpoint writes; they can be re-ordered as necessary by lower layers in the storage stack to realize the benefits of low-level I/O scheduling. Further, by grouping all writes into a single, large transaction, writes are effectively committed in program order: if a write to $f_{1}$ occurs before a write to $f_{2}$, they will either be committed together (in the same transaction), or the write to $f_{2}$ will commit later; never will $f_{2}$ commit before $f_{1}$. We discuss ext 4 journaling in more detail in the next section.

Unfortunately, total write ordering, as provided with data journaling, exacts a high performance cost: each data item must be written twice, thus halving disk bandwidth for some workloads. For this reason, most journaling file systems only journal metadata, maintaining file-system crash consistency but losing ordering among application writes. What would be ideal is the performance of metadata-only journaling combined with the ordering guarantees provided by full data journaling.

However, even if an efficient journaling mechanism is used, it does not avoid overheads due to false dependence. To address this problem, we believe a new abstraction is needed, which enables the file system to separate update orderings across different applications. Within an application, we believe that false dependence is rare and does not typically arise.

Thus, we are left with two open questions. Can a metadata-only journaling approach be adopted that maintains order but with high performance? Second, can a new abstraction eliminate false dependence? We answer these questions in the affirmative with the design and implementation of ccfs.

\section{CRASH-CONSISTENT FILE SYSTEM}

In this section, we describe ccfs, a file system that embraces application-level crash consistency. Ccfs has two goals corresponding to our two hypotheses in Section 2.1: first, it aims to preserve the program order of updates and provide weak atomicity; second, it aims to achieve performance 
similar to widely-used re-ordering file systems. To satisfy these goals, we derive ccfs from the ext4 file system. Ext4 is widely used, includes many optimizations that allow it to perform efficiently in real deployments, and includes a journaling mechanism for internal file-system consistency. In ccfs, we extend ext4's journaling to preserve the required order and atomicity in an efficient manner without affecting the optimizations already present in ext4.

The key idea in ccfs is to separate each application into a stream and maintain order only within each stream; writes from different streams are re-ordered for performance. This idea has two challenges: metadata structures and the journaling mechanism need to be separated between streams, and order needs to be maintained within each stream efficiently. Ccfs should solve both without affecting existing file-system optimizations. In this section, we first explain ext4's journaling mechanism (Section 3.1), then the streams abstraction (Section 3.2), how streams are separated (Section 3.3) and how order is maintained within a stream (Section 3.4), and our implementation (Section 3.5). We finally discuss how applications can practically start using the streams abstraction (Section 3.6).

\subsection{Journaling in Ext4}

To maintain file-system metadata consistency, ext4 requires the atomicity of sets of metadata updates (e.g., all metadata updates involved in creating a file) and an order between these sets of updates [6, 25]. Ext4 uses an optimized journaling technique for this purpose. Specifically, the journaling occurs at block granularity, batches multiple sets of atomic metadata updates (delayed logging [12]), uses a circular journal, and delays forced checkpointing until necessary. The blockgranularity and circular aspects prove to be a challenge for adoption in ccfs, while delayed logging and checkpointing are important optimizations that ccfs needs to retain. We now briefly explain these techniques of ext4 journaling.

Assume the user performs a metadata operation (such as creating a file), causing ext 4 to modify metadata structures in the file-system blocks $b_{1}, b_{2}, b_{3}$. Ext 4 associates $b_{1}, b_{2}, b_{3}$ with an in-memory data structure called the running transaction, $T_{i}$. Instead of immediately persisting $T_{i}$ when the metadata operation completes, ext4 waits for the user to perform more operations; when this happens, the resulting set of block modifications are also associated with $T_{i}$ (i.e., delayed logging). Periodically, ext4 commits the running transaction, that is, writes the updated contents of all the associated blocks of $T_{i}$ and some bookkeeping information to an on-disk journal. When $T_{i}$ starts committing, a new running transaction $\left(T_{i+1}\right)$ is created to deal with future metadata operations. Thus, ext4 always has one running transaction, and at most one committing transaction. Once $T_{i}$ finishes committing, its blocks can be written to their actual locations on disk in any order; this is usually done by Linux's page-flushing daemon in an optimized manner.

If a crash happens, then after rebooting, ext4 scans each transaction written in the journal file sequentially. If a transaction is fully written, then the blocks recorded in that transaction are propagated to their actual locations on disk; if not, then ext4 stops scanning the journal. Thus, the atomicity of all block updates within each transaction is maintained. Maintaining atomicity also implicitly maintains order within a transaction, while the sequential scan of the journal maintains order across transactions.

The on-disk journal file is circular: after the file reaches a maximum size, committed transactions in the tail of the journal are freed (i.e., checkpointed) and that space is reused for recording future transactions. Ext4 ensures that before a transaction's space is reused, the blocks contained in it are first propagated to their actual locations (if the page-flushing mechanism had not yet propagated them). Ext4 employs techniques that coalesce such writebacks. For example, consider that a block recorded in $T_{i}$ is modified again in $T_{j}$; instead of writing back the version of the block recorded in $T_{i}$ and $T_{j}$ separately, ext 4 simply ensures that $T_{j}$ is committed before $T_{i}$ 's space is reused. Since the 
more recent version (in $T_{j}$ ) of the block will be recovered on a crash without violating atomicity, the earlier version of the block will not matter. Similar optimizations also handle situations where committed blocks are later unreferenced, such as when a directory gets truncated.

For circular journaling to work correctly, ext4 requires a few invariants. One invariant is of specific interest in ccfs: the number of blocks that can be associated with a transaction is limited by a threshold. To enforce the limit, before modifying each atomic set of metadata structures, ext4 first verifies that the current running transaction (say, $T_{i}$ ) has sufficient capacity left; if not, ext4 starts committing $T_{i}$ and uses $T_{i+1}$ for the modifications.

\subsection{Streams}

Ccfs introduces a new abstraction called the stream. We now first explain the stream abstraction, and then the API for the abstraction.

Stream Abstraction. In ccfs, each application usually corresponds to a single stream. Writes from different streams are re-ordered for performance, while order is preserved within streams for crash consistency. We define the stream abstraction such that it can be easily used in common workflows; as an example, consider a text file $f 1$ that is modified by a text editor while a binary file $f 2$ is downloaded from the network, and they are both later added to a VCS repository. Initially, the text editor and the downloader must be able to operate on their own streams (say, $A$ and $B$, respectively), associating $f 1$ with $A$ and $f 2$ with $B$. Note that there can be no constraints on the location of $f 1$ and $f 2$ : the user might place them in the same directory. Moreover, the VCS should then be able to operate on another stream $C$, using $C$ for modifying both $f 1$ and $f 2$. In such a scenario, the stream abstraction should guarantee the order required for crash consistency, while allowing enough reordering for the best performance possible.

Hence, in ccfs, streams are transient and are not uniquely associated with specific files or directories: a file that is modified in one stream might be later modified in another stream. However, because of such flexibility, while each stream can be committed independently without being affected by other streams, it is convenient if the stream abstraction takes special care when two streams perform operations that affect logically related data. For example, consider a directory that is created by stream $A$, and a file that is created within the directory by stream $B$; allowing the file creation to be re-ordered after the directory creation (and recovering the file in a lost+found directory on a crash) might not make logical sense from an application's perspective. Hence, when multiple streams perform logically related operations, the file system takes sufficient care so the temporal order between those operations is maintained on a crash.

We loosely define the term related such that related operations do not commonly occur in separate streams within a short period of time; if they do, the file system might perform inefficiently. For example, separate directory entries in a directory are not considered related (since it is usual for two applications to create files in the same directory), but the creation of a file is considered related to the creation of its parent. Figure 2(i) illustrates two logically related streams, where stream $A$ first creates a directory ( $f o o$ ), after which stream $B$ creates a file (bar) inside foo. When stream $B$ calls an fsync on bar, the creat of bar has to be flushed, which requires flushing the mkdir of its parent directory, ultimately requiring the 1GB write to $f$ in $A$ also to be flushed. In Figure 2(ii), streams $A$ and $B$ are unrelated; thus, neither the mkdir of foo nor the $1 \mathrm{~GB}$ write need to be flushed when $B$ calls an fsync on bar. We leave the exact definition of logically related operations to file systems that implement streams, instead of the stream abstraction itself; in Section 3.5, we discuss the set of logically related operations in ccfs.

Although each application (perhaps containing multiple threads and processes) usually corresponds to a single stream, the abstraction allows for more flexibility; Figure 3 shows different ways 


\begin{tabular}{|c|c|c|c|}
\hline $\begin{array}{c}\text { Stream } A \\
\operatorname{write}(f, 1 \mathrm{~GB}) \\
\operatorname{mkdir}(f \circ o)\end{array}$ & $\begin{array}{l}\operatorname{creat}(\text { foo/bar); } \\
\text { write(bar , 'a'); } \\
\text { fsync(bar); }\end{array}$ & \begin{tabular}{l}
\multicolumn{1}{c}{$\operatorname{Stream} A$} \\
write $(f, 1 G B)$ \\
mkdir $(f \circ o)$
\end{tabular} & $\begin{array}{l}\text { creat(bar); } \\
\text { write(bar, 'a'); } \\
\text { fsync(bar); }\end{array}$ \\
\hline
\end{tabular}

Fig. 2. Related and non-related operations across streams. Panel (i) shows a scenario where streams $A$ and $B$ are related: $B$ creates a file barinside the directory foocreated in stream $A$. In panel (ii), the streams perform similar operations but are unrelated and can commit independently.

in which streams can be used and shared by applications. In addition to the usual case (Figure 3(a)), each thread in the application can use a different stream (Figure 3(b)) if necessary, a single thread can switch between different streams (Figure 3(c)), and multiple applications can share a stream (Figure 3(d)). We next explain the interface that allows such flexibility.

Stream API. Table 2 shows the stream API. The setstream(s) call creates a new stream (if not already existing) and associates the current thread with the stream s. All future updates in that thread will be assigned to stream s; when forking (a process or thread), a child will adopt the stream of its parent.

With the default setstream(s) call, all fsync calls issued in the stream will be obeyed. Thus, in this mode, applications will observe similar performance to the case where they run on a re-ordering file system. However, the in-order update semantics of ccfs results in better consistency guarantees.

To improve performance, all unnecessary fsync calls can be ignored. An fsync call is unnecessary if it only intends to enforce ordering but not durability. Since ccfs inherently provides the required ordering, such fsync calls can be eliminated from the application. To ease this elimination, the setstream() call also takes a flag called IGNORE_FSYNC specified as part of the optional flags parameter. If the IGNORE_FSYNC flag is set, then all fsync calls in the stream will be ignored, improving performance while maintaining correctness.

However, some fsync calls may be required to enforce durability not just ordering. For example, while SQLite may issue several fsync calls to order updates, it also issues fsync calls at a few places to enforce durability (e.g., transaction commit). To enforce durability where required, the streamsync() call can be utilized; this call flushes all updates in the current stream. Identifying places where streamsync() calls are required is straightforward. Developers and users can add these calls at points where durability is desired (e.g., transaction commit points in SQLite). In addition, tools such as Alice can help identify where the streamsync() calls are required.

Figure 4 shows how the write-ahead logging protocol (shown earlier in Figure 1) can be made crash consistent atop ccfs. Part (i) shows a variation of the original protocol that works on ext3 and ext 4 but might not work atop file systems such as ext2 or btrfs [38]. Part (ii) shows the changes required to make this protocol crash consistent atop ccfs while providing the same performance as the one shown in part (i). To improve performance atop ccfs, one can use the IGNORE_FSYNC flag and issue a streamsync() call for durability at the end of the protocol as shown in part (iii). Note that issuing the streamsync() call at any point after the journal write ensures durability.

The stream API also provides a getstream() call that is used, for example, to find if the current process is operating on the init stream (explained in Section 3.6) or a more specific stream. The API usage is further explained in Section 3.6. 


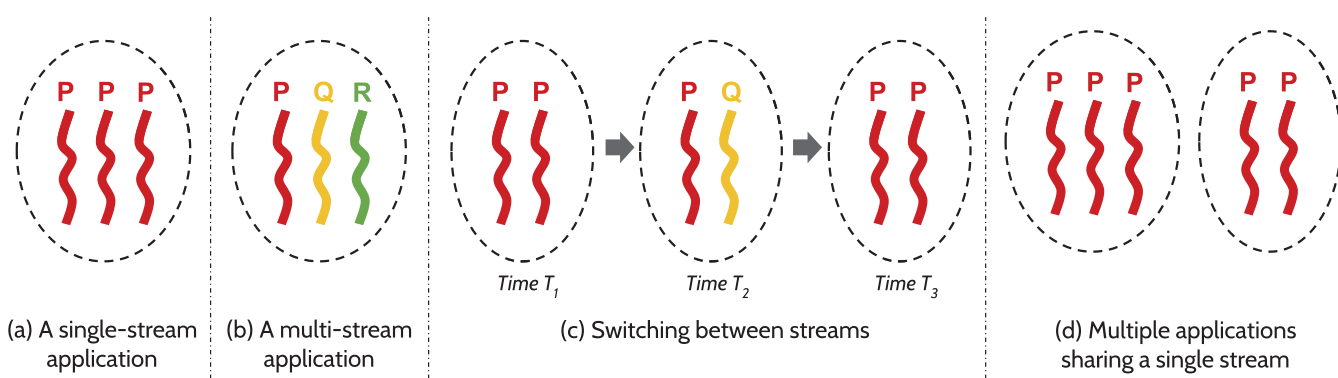

Fig. 3. Using Streams. The figure shows different ways in which streams can be used and shared. A dotted oval represents an application consisting of multiple threads (or processes), and each wavy line within the oval represents a thread. Panel (a) shows the case where all threads of an application share a single stream, P. Panel (b) shows the case where each thread of an application uses a different stream. Panel (c) shows a scenario where a thread switches between two streams $P$ and $Q$. Panel (d) shows the case where multiple applications share the same stream, $P$.

Table 2. Stream API

\begin{tabular}{c|c}
\hline Call & Description \\
\hline setstream(id, flags) & $\begin{array}{c}\text { Creates the stream id and associates the calling thread with } \\
\text { it. The flags parameter is optional. If the IGNORE_FSYNC flag } \\
\text { is set, then all fsync calls issued in the stream will be ignored. }\end{array}$ \\
\hline getstream() & $\begin{array}{c}\text { Gets the stream identifier of the calling thread. This call is } \\
\text { used to check whether a stream is already set. }\end{array}$ \\
\hline streamsync() & Flushes all updates in the stream. \\
\hline
\end{tabular}

\subsection{Separating Multiple Streams}

In ccfs, the basic idea used to separately preserve the order of each stream is simple: ccfs extends the journaling technique to maintain multiple in-memory running transactions, one corresponding to each stream. Whenever a synchronization system call (such as fsync) is issued, only the corresponding stream's running transaction is committed. All modifications in a particular stream are associated with that stream's running transaction, thus maintaining order within the stream (optimizations regarding this are discussed in the next section).

Using multiple running transactions poses a challenge: committing one transaction without committing others (i.e., re-ordering between streams) inherently re-orders the metadata modified across streams. However, internal file-system consistency relies on maintaining a global order between metadata operations; indeed, this is the original purpose of ext4's journaling mechanism. It is hence important that metadata modifications in different streams be logically independent and be separately associated with their running transactions. We now describe the various techniques that ccfs uses to address this challenge while retaining the existing optimizations in ext 4.

3.3.1 Hybrid-Granularity Journaling. The journaling mechanism described previously (Section 3.1) works at block-granularity: entire blocks are associated with running transactions, and committing a transaction records the modified contents of entire blocks. Ccfs uses hybridgranularity journaling, where byte-ranges (instead of entire blocks) are associated with the running transaction, but transactional commits and checkpointing still happen at the granularity of blocks.

Ccfs requires byte-granularity journaling, because separate metadata structures modified by different streams might exist in the same file-system block. For example, a single block can contain 


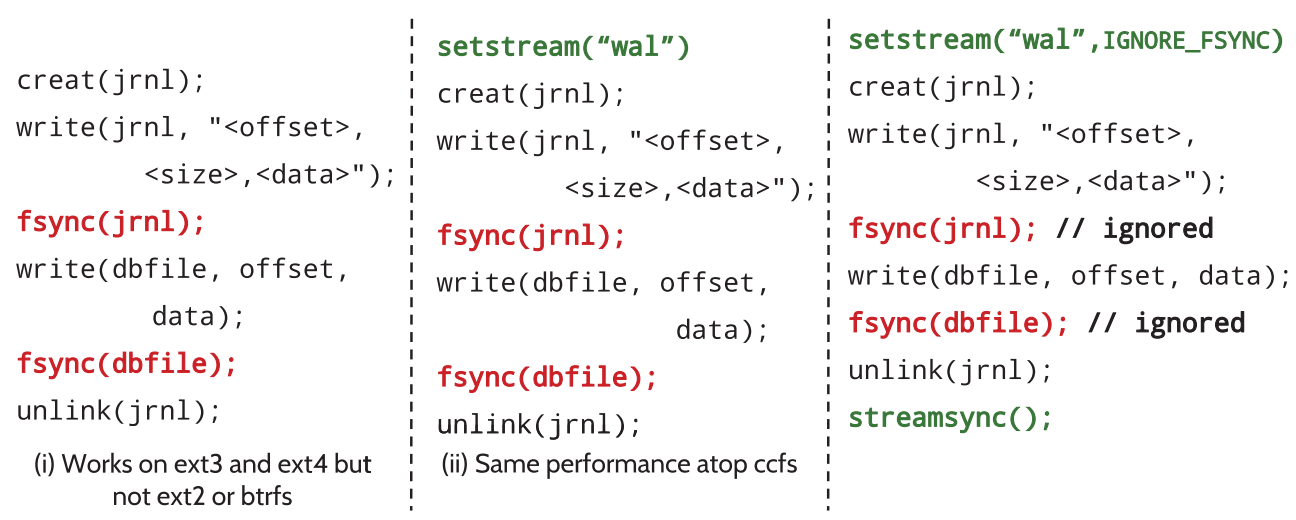

Fig. 4. Crash-consistent write-ahead logging protocol atop ccfs. Panel (i) shows a slight modification of Figure 1 that works correctly on some versions of ext4 and ext3, but might not on other file systems (such as ext2 or btrfs). Panel (ii) shows the change (a single setstream() call) required to the previous figure for guaranteeing correctness using streams while achieving similar performance. Panel (iii) shows the changes required to improve performance using streams.

the inode structure for two files used by different applications; in block-granularity journaling, it is not possible to associate the inodes with the separate running transactions of two different streams.

Block-granularity journaling allows many optimizations that are not easily retained in bytegranularity. A major optimization affected in ext 4 is data coalescing during checkpoints: even if multiple versions of a block are committed, only the final version is sent to its in-place location. Since the Linux buffer cache and storage devices manage data at block granularity, such coalescing becomes complicated with a byte-granularity journal.

To understand hybrid-granularity journaling, consider the example illustrated in Figure 5. In this example, block $X$ initially contains the bytes $\left\langle a_{0} b_{0}\right\rangle$. Before allowing any writes, ccfs makes an in-memory copy (say, $X^{0}$ ) of the initial version of the block. Let the first byte of $X$ be modified by stream $A$ into $a_{1}$; ccfs will associate the byte range $X_{0-0}$ with the running transaction $T_{A}$ of stream $A\left(X_{i-j}\right.$ denotes the $i$ th to $j$ th bytes of block $\left.X\right)$, thus following byte-granularity. Let stream $B$ then modify the second byte into $b_{1}$, associating $X_{1-1}$ with $T_{B}$; the final in-memory state of $X$ will be $\left\langle a_{1} b_{1}\right\rangle$. Now, assume the user calls fsync in stream $B$, causing $T_{B}$ to commit (T $T_{A}$ is still running). Ccfs converts $T_{B}$ into block-granularity for the commit, by super-imposing the contents of $T_{B}$ (i.e., $X_{1-1}$ with the content $b_{1}$ ) on the initial versions of their blocks (i.e, $X^{0}$ with content $\left\langle a_{0} b_{0}\right\rangle$ ), and committing the result (i.e., $\left\langle a_{0} b_{1}\right\rangle$ ). When $T_{B}$ starts committing, it updates $X^{0}$ with the value of $X$ that it is committing. If the user then calls fsync in $A$, then $X_{0-0}$ is super-imposed on $X^{0}\left(\left\langle a_{0} b_{1}\right\rangle\right)$, committing $\left\langle a_{1} b_{1}\right\rangle$.

Thus, hybrid-granularity journaling performs in-memory logging at byte-granularity, allowing streams to be separated; the delayed-logging optimization of ext4 is unaffected. Commits and checkpoints are block-granular, thus preserving delayed checkpointing.

3.3.2 Delta Journaling. In addition to simply associating byte ranges with running transactions, ccfs allows associating the exact changes performed on a specific byte range (i.e., the deltas). This technique, which we call delta journaling, is required when metadata structures are actually shared between different streams (as opposed to independent structures sharing the same block). For 


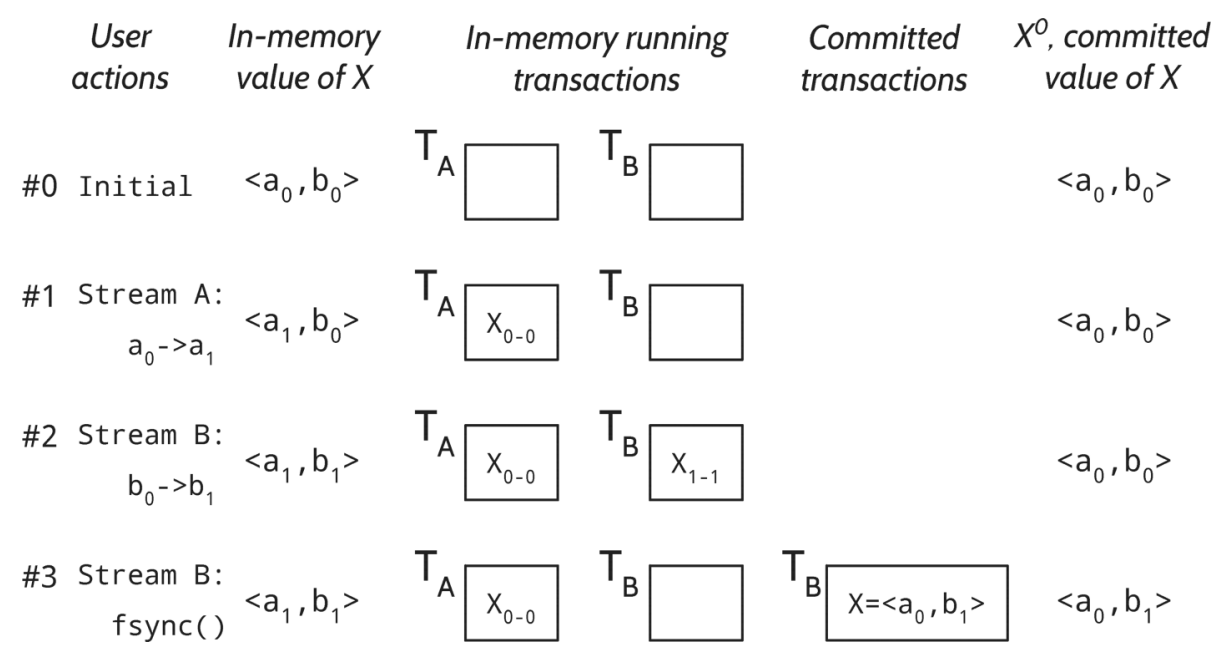

Fig. 5. Hybrid-granularity Journaling. Timeline showing hybrid-granularity journaling in ccfs. Block X initially contains the value $\left\langle a_{0}, b_{0}\right\rangle, T_{A}$ and $T_{B}$ are the running transactions of streams $A$ and $B$; when $B$ commits, $X$ is recorded at the block level on disk.

example, consider a metadata structure tracking the total free space in the file system: all streams need to update this metadata structure.

Delta journaling in ccfs works as follows. Assume that the byte range $X_{1-2}$ is a shared metadata field storing an integer, and that stream $A$ adds $i$ to the field and stream $B$ subtracts $j$ from the field. Ccfs associates the delta $\left\langle X_{1-2}:+i\right\rangle$ to the running transaction $T_{A}$ and the delta $\left\langle X_{1-2}:-j\right\rangle$ to the running $T_{B}$. When a transaction commits, the deltas in the committing transaction are imposed on the initial values of their corresponding byte ranges, and then the results are used for performing the commit. In our example, if $X_{1-2}$ initially had the value $k$, and stream $B$ committed, the value $(k-$ $j$ ) will be recorded for the byte range during the commit; note that hybrid-granularity journaling is still employed, that is, the commit will happen at block-granularity.

In ext4, shared metadata structures requiring delta journaling are the free inode count and the free block count, which concern the global state across the file system. Delta journaling is also needed for the nlink and the modification time fields of directory inodes, since multiple streams can modify the same directory.

3.3.3 Pointer-less Data Structures. Metadata in file systems often use data structures such as linked lists and trees that contain pointers, and these cause metadata operations in one stream to update pointers in structures already associated with another stream. For example, deleting an entry in a linked list will require updating the next pointer of the previous entry, which might be associated with another stream. Ccfs eliminates the need to update pointers across streams by adopting alternative data structures for such metadata.

Ext4 has two metadata structures that are of concern: directories and the orphan list. Directories in ext4 have a structure similar to linked lists, where each entry contains the relative byte-offset for the next entry. Usually, the relative offset recorded in a directory entry is simply the size of the entry. However, to delete a directory entry $d_{i}$, ext 4 adds the size of $d_{i}$ to the offset in the previous entry $\left(d_{i-1}\right)$, thus making the previous entry point to the next entry $\left(d_{i+1}\right)$ in the list. To make directories pointer-less, ccfs replaces the offset in each entry with a deleted bit: deleting an entry sets the bit. The insert and scan procedures are modified appropriately; for example, the insert 
procedure recognizes previously deleted entries in the directory and uses them for new entries if possible.

The orphan list in ext4 is a standard linked list containing recently freed inodes and is used for garbage collecting free blocks. The order of entries in the list does not matter for its purposes in ext4. We convert the orphan list into a pointer-less structure by substituting it with an orphan directory, thus reusing the same data structure.

3.3.4 Order-Less Space Reuse. Ccfs carefully manages the allocation of space in the file system such that re-ordering deallocations between streams does not affect file-system consistency. For example, assume stream $A$ deletes a file and frees its inode, and stream $B$ tries to create a file. The allocation routines in ext 4 might allocate to $B$ the inode that was just freed by $A$. However, if $B$ commits before $A$, and then a crash occurs, the recovered state of the file system will contain two unrelated files assigned the same inode.

Ext4 already handles the situation for block allocation (for reasons of security) by reusing blocks only after the transaction that frees those blocks has fully committed. In ccfs, we extend this solution to both inode and directory-entry reuse. Thus, in our example, $B$ will reuse $A$ 's freed inode only if $A$ has already been committed.

\subsection{Maintaining Order Within Streams}

We saw in the previous section how to separate dependencies across independent streams; we now focus on ordering the updates within the same stream. Ext4 uses metadata-only journaling: ext4 can re-order file appends and overwrites. Data journaling, that is, journaling all updates, preserves application order for both metadata and file data, but significantly reduces performance, because it often writes data twice. A hybrid approach, selective data journaling (SDJ) [6], preserves order of both data and metadata by journaling only overwritten file data; it only journals the block pointers for file appends. Since modern workloads are mostly composed of appends, SDJ is significantly more efficient than journaling all updates.

We adopt the hybrid SDJ approach in ccfs. However, the approach still incurs noticeable overhead compared to ext4's default journaling under practical workloads, because it disables a significant optimization, delayed allocation. In our experiments with ext4 (Linux 3.13) on a Toshiba MK1665GSX 160GB HDD, the createfiles benchmark results in 8,795ops/s with delayed allocation, and $7,730 \mathrm{ops} / \mathrm{s}$ without (12\% overhead).

Without delayed allocation, whenever an application appends to files, data blocks are allocated and block pointers are assigned to the files immediately, as shown in the second column of Figure 6. With delayed allocation (third column), the file system does not immediately allocate blocks; instead, allocations for multiple appends are delayed and done together. For order to be maintained within a stream, block pointers need to be assigned immediately (for example, with SDJ, only the order of allocations is preserved across system crashes): naive delayed allocation inherently violates order.

Ccfs uses a technique that we call order-preserving delayed allocation to maintain program order while allowing delayed allocations. Whenever a transaction $T_{i}$ is about to commit, all allocations (in the current stream) that have been delayed so far are performed and added to $T_{i}$ before the commit; further allocations from future appends by the application are assigned to $T_{i+1}$. Thus, allocations are delayed until the next transaction commit, but not across commits. Since order is maintained within $T_{i}$ via the atomicity of all operations in $T_{i}$, the exact sequence in which updates are added to $T_{i}$ does not matter, and thus the program order of allocations is preserved.

However, the running transaction's size threshold poses a challenge: at commit time, what if we cannot add all batched allocations to $T_{i}$ ? Ccfs solves this challenge by reserving the space required 


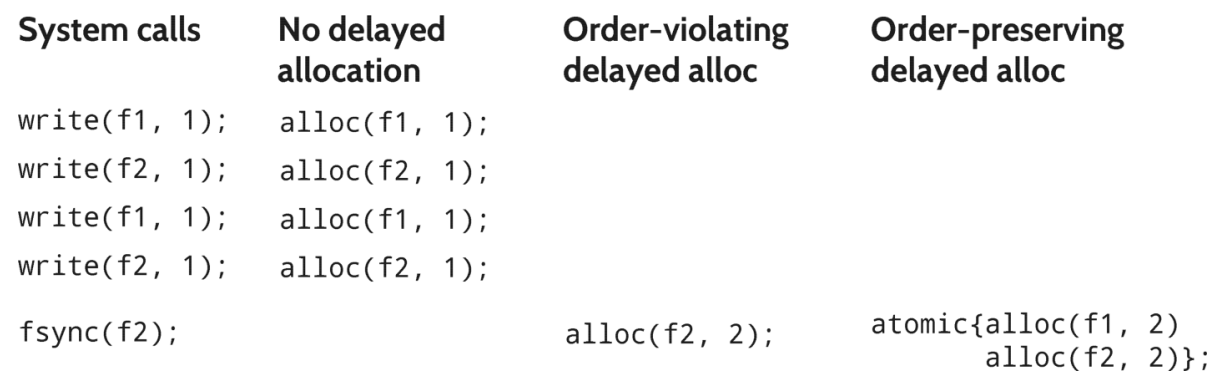

Fig. 6. Order-preserving Delayed Allocation. Timeline of allocations performed, corresponding to a system-call sequence.

for allocations when the application issues the appends. Order-preserving delayed allocation thus helps ccfs achieve ext4's performance while maintaining order. For the createfiles benchmark, the technique achieves $8,717 \mathrm{ops} / \mathrm{s}$ in ccfs, and thus performs similar to the default configuration of ext4 (8,795ops/s).

\subsection{Implementation}

Ccfs changes 4,500 lines of source code (original ext4 total: 50,000 lines). Of these, preserving order (Section 3.4) required only 500 lines while implementing multiple streams (Section 3.3) was more complicated and involved the rest of the changes. Overall, most of the changes (3,000 lines) are related to the journaling code within ext4. We now describe our implementation.

Related Operations Across Streams. The current version of ccfs considers the following operations as logically related: modifying the same regular file, explicitly modifying the same inode attributes (such as the owner attribute), updating (creating, deleting, or modifying) directory entries of the same name within a directory, and creating a directory and any directory entries within that directory. To understand how ccfs maintains temporal ordering between related operations from different streams, consider that stream $A$ first performs operation $O_{A}$ at time $t_{1}$ and stream $B$ then performs a related operation $O_{B}$ at $t_{2}$. If stream $A$ gets committed between $t_{1}$ and $t_{2}$ (either due to an fsync or a periodic background flush), then the required temporal order is already maintained, since $O_{A}$ is already on disk before $O_{B}$ is performed. If not, then ccfs temporarily merges the streams together and treats them as one, until the merged streams get committed to disk; the streams are then separated and allowed to proceed independently.

Maintaining Order Within Streams. An implementation challenge for order-preserving delayed allocation is that the allocations need to be performed when a transaction is about to commit, but before the actual committing starts. We satisfy these requirements without much complexity by performing the allocations in the T_LOCKED state of the transaction, a transient state in the beginning of every commit when all file-system updates are blocked. A more efficient implementation could perform these allocations before the T_LOCKED state.

To correctly maintain the order of file updates, SDJ requires careful handling when data is both appended and overwritten on the same block. For example, consider an append when $T_{i}$ was running and an overwrite when $T_{i}$ is committing (when $T_{i+1}$ is running); to maintain order, two versions of the block must be created in memory: the old version (that does not contain the overwrite) must be used as part of $T_{i}$ 's commit, and the new version must be journaled in $T_{i+1}$. Ccfs handles these cases correctly. 


\subsection{Discussion}

We now discuss how we expect applications to use streams. Overall, the abstraction is flexible: while we expect most applications to use a single stream, if needed, applications can also use separate streams for individual tasks, or multiple applications can share a single stream. In the current version of ccfs, the init process is assigned an init stream; hence, all applications inherit this stream by default. We expect most applications whose write performance is user visible to issue a single setstream() call in the beginning of the application (but to not make any other code changes). Thus, applications by default will have improved crash consistency, and applications issuing setstream() will have both improved consistency and high performance. If so desired, then applications can also significantly improve their performance (while maintaining consistency) by first setting the IGNORE_FSYNC flag and removing any 0_SYNC flags, and issuing streamsync() calls only when durability is actually desired.

\section{EVALUATION}

In our evaluation, we first confirm our ordering hypothesis by answering the following question: does the ordering guarantee provided by ccfs improve application crash consistency?

Next, we confirm our efficiency hypothesis by answering the following questions:

- Does ccfs effectively use streams to eliminate the overhead of write dependencies?

- How does ccfs perform in standard file system benchmarks run in a single stream?

- What is the performance effect of maintaining order on real applications?

Finally, we analyze how much developer effort is needed to make an application crash consistent atop ccfs.

We performed a set of experiments to answer these questions. For the experiments, we use an Intel Core 2 Quad Processor Q9300 with 4GB of memory running Linux 3.13, with either an SSD (Samsung 840 EVO 500GB) or a HDD (Toshiba MK1665GSX 160GB).

\subsection{Reliability}

We first examine whether the in-order semantics provided by ccfs improves application crash consistency compared to the widely used ext4 file system (which re-orders writes). We follow a model-based testing strategy to check application consistency on both file systems using the Alice tool [38]. The tool records the system-call trace for a given application workload, and then uses a file-system model to reproduce the possible set of file-system states if a system crash occurs. We configured Alice with the models of ext4 (model provided with the tool) and ccfs (system calls are weakly atomic and in-order). We tested five applications previously reported [38] to exhibit crash inconsistencies on ext4: LevelDB, SQLite, Git, Mercurial, and ZooKeeper. We use workloads similar to the previous study, but newer versions of the applications; we do not check durability in Git and Mercurial, since they never call fsync.

The results of our testing are shown in Table 3(a). Ext4 results in multiple inconsistencies: LevelDB fails to maintain the order in which key-value pairs are inserted, Git and Mercurial can result in repository corruption, and ZooKeeper may become unavailable. With ccfs, the only inconsistencies were with Mercurial. These inconsistencies are exposed on a process crash with any file system, and therefore also occur during system crashes in ccfs; they result only in dirstate corruption, which can be manually recovered from and is considered to be of minor consequence [29]. Thus, our model-based testing reveals that applications are significantly more crash consistent on ccfs than ext4. 
Table 3. Consistency Testing

\begin{tabular}{r|c|c} 
Application & ext4 & ccfs \\
\hline LevelDB & 1 & 0 \\
\hline SQLite-Roll & 0 & 0 \\
\hline Git & 2 & 0 \\
\hline Mercurial & 5 & 2 \\
\hline ZooKeeper & 1 & 0 \\
\hline
\end{tabular}

(a) Vulnerabilities found

\begin{tabular}{c|r|c|c}
\multicolumn{2}{c|}{ Application } & ext4 & ccfs \\
\hline \multirow{2}{*}{ LevelDB } & Images & $158 / 465$ & $427 / 427$ \\
& Time (s) & $24.31 / 30$ & $30 / 30$ \\
\hline \multirow{2}{*}{ Git } & Images & $84 / 112$ & $96 / 96$ \\
& Time (s) & $9.95 / 40$ & $40 / 40$ \\
\hline
\end{tabular}

(b) Consistent post-reboot disk states produced by $\mathrm{BoB}$

The first table shows the results of model-based testing using Alice, and the second shows experimental testing with BoB. Each vulnerability reported in the first table is a location in the application source code that has to be fixed. The Imagesrows of the second table show the number of disk images reproduced by the BoB tool that the application correctly recovers from; the Timerows show the time window during which the application can recover correctly from a crash ( $x / y: x$ is the time window, $y$ is the total workload runtime, both in seconds). For Git, we consider the default configuration instead of a safer configuration with bad performance (Section 4.4).

We used the BoB tool [38] to test whether our implementation of ccfs maintains weak atomicity and ordering, that is, whether the implementation reflects the model used in the previous testing. $\mathrm{BoB}$ records the block-level trace for an application workload running on a file system, and reproduces a subset of disk images possible if a crash occurs. BoB generates disk images by persisting blocks in and out of order; each image corresponds to a time window during the runtime where a crash will result in the image. These windows are used to measure how much time the application remains consistent.

We used Git and LevelDB to test our implementation and compare it with ext4; both have crash vulnerabilities exposed easily on a re-ordering file system. Table 3(b) shows our results. With ext4, both applications can easily result in inconsistency. LevelDB on ext4 is consistent only on 158 of the 465 images reproduced; a system crash can result in being unable to open the datastore after reboot, or violate the order in which users inserted key-value pairs. Git will not recover properly on ext4 if a crash happens during 30.05s of the 40s runtime of the workload. With ccfs, we were unable to reproduce any disk state in which LevelDB or Git are inconsistent. We conclude that our implementation provides the desired properties for application consistency.

Thus, our results show that the in-order semantics provided by ccfs noticeably improves the state of application crash consistency, confirming our ordering hypothesis. We next validate our efficiency hypothesis by evaluating whether ccfs provides this improved consistency with good performance.

\subsection{Multi-Stream Benefits}

Maintaining order causes write dependence during fsync calls and imposes additional overheads, since each fsync call must flush all previous dirty data. In the simplest case, this results in additional fsync latency; it can also prevent writes from being coalesced across fsync calls when data is overwritten, and prevent writes from being entirely avoided when the previously written data is deleted. We now evaluate if using separate streams in ccfs prevents these overheads.

We devised three microbenchmarks to study the performance effects of preserving order. The append microbenchmark appends a large amount of data to file $A$, then writes 1 byte to file $B$ and calls fsync on $B$; this stresses the fsync call's latency. The truncate benchmark truncates file $A$ after calling fsync while overwrite overwrites $A$ after the fsync; these benchmarks stress whether or not writes are avoided or coalesced. 
Table 4. Single-fsync Experiments

\begin{tabular}{c|c|c|c|c}
\hline $\begin{array}{c}\text { Micro } \\
\text { Benchmark }\end{array}$ & $\begin{array}{c}\text { File } \\
\text { system }\end{array}$ & $\begin{array}{c}\text { fsync } \\
\text { latency (s) }\end{array}$ & $\begin{array}{c}\text { fsync } \\
\text { written (MB) }\end{array}$ & $\begin{array}{c}\text { Total written } \\
\text { (MB) }\end{array}$ \\
\hline \multirow{3}{*}{ Append } & ext4 & 0.08 & 0.03 & 100.19 \\
& ccfs-1 & 1.28 & 100.04 & 100.18 \\
& ccfs-2 & 0.08 & 0.03 & 100.20 \\
\hline \multirow{3}{*}{ Truncate } & ext4 & 0.07 & 0.03 & 0.18 \\
& ccfs-1 & 1.28 & 100.04 & 100.21 \\
& ccfs-2 & 0.05 & 0.03 & 0.20 \\
\hline \multirow{3}{*}{ Overwrite } & ext4 & 0.08 & 0.03 & 100.19 \\
& ccfs-1 & 1.27 & 100.04 & 300.72 \\
& ccfs-2 & 0.07 & 0.03 & 100.20 \\
\hline
\end{tabular}

fsynclatencies in the first column correspond to the data written by the fsyncshown in the second column on HDD, while the total data shown in the third column affects the available device bandwidth and hence performance in more realistic workloads. In the ccfs- 1 configuration, updates to both files $A$ and $B$ are performed in a single stream. In the ccfs- 2 configuration, Modifications to $A$ and $B$ are performed in two separate streams. The numbers reported are the average over 10 runs.

We use two versions of each benchmark. In the simpler version, we write $100 \mathrm{MB}$ of data in file $A$ and measure the latency of the fsync call and the total data sent to the device. In another version, a foreground thread repeatedly writes $B$ and calls fsync every $5 \mathrm{~s}$; a background thread continuously writes to $A$ at $20 \mathrm{MB} / \mathrm{s}$, and may truncate $A$ or overwrite $A$ every $100 \mathrm{MB}$, depending on the benchmark. The purpose of the multi-fsync version is to understand the distribution of fsync latencies observed in such a workload.

We ran the benchmarks on three file-system configurations: ext4, which re-orders writes and does not incur additional overheads, ccfs using a single stream (ccfs-1), and ccfs with modifications of $A$ and $B$ in separate streams (ccfs-2). Table 4 and Figure 7 show our results.

For the append benchmark, in ext4, the fsync completes quickly in $0.08 \mathrm{~s}$, since it flushes only $B$ 's data to the device. In ccfs-1, the fsync sends $100 \mathrm{MB}$ and takes $1.28 \mathrm{~s}$, but ccfs-2 behaves like ext4, since $A$ and $B$ are modified in different streams. Repeated fsync follows the same trend: most fsync calls are fast in ext 4 and ccfs- 2 but often take more than 1 s in ccfs- 1 . A few fsync calls in ext 4 and ccfs- 2 are slow due to interference from background activity by the page-flushing daemon and the periodic journal commit.

With truncates, ext 4 and ccfs- 2 never send file $A$ 's data to disk, but ccfs- 1 sends the $100 \mathrm{MB}$ during fsync, resulting in higher latency and more disk writes. Most repeated fsync calls in ext4 and ccfs- 2 are fast, as expected; they are slow in ccfs-1, but still quicker than the append benchmark, because the background thread would have just truncated $A$ before some of the fsync.

With overwrites, in both ext4 and ccfs-2, only the final version of $A$ 's data reaches the disk: in ccfs-2, SDJ (selective data journaling) considers the second modification of $A$ an append, because the first version of $A$ is not yet on disk (this still maintains order). In ccfs- 1 , the first version is written during the fsync, and then the second version (overwrite) is both written to the journal and propagated to its actual location, resulting in $300 \mathrm{MB}$ of total disk writes. Repeated fsync calls are slow in ccfs- 1 but quicker than previous benchmarks because of fewer disk seeks: with this version of the benchmark, since $A$ is constantly overwritten, data is only sent to the journal in ccfs- 1 and is never propagated to its actual location. 


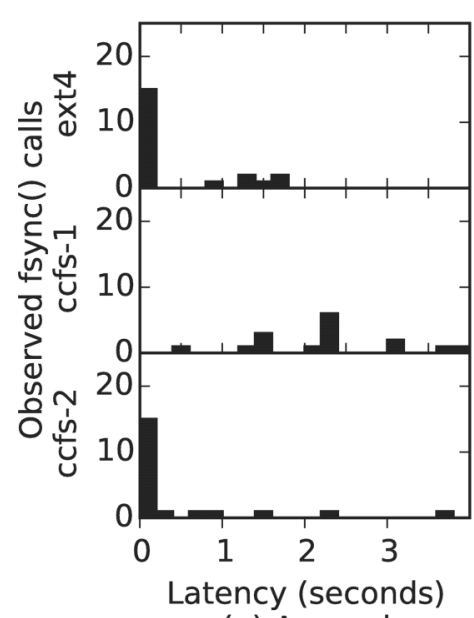

(a) Append

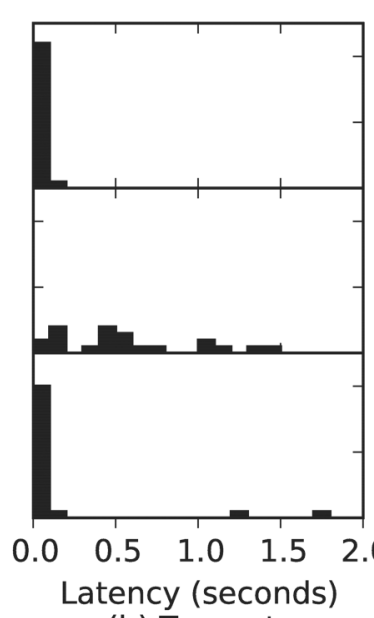

(b) Truncate

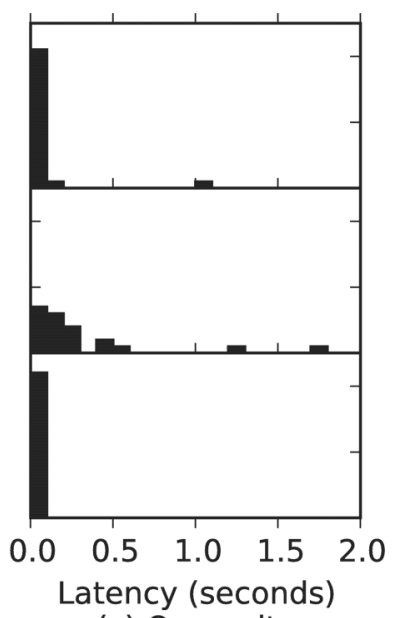

(c) Overwrite

Fig. 7. Repeated fsync Experiments. Histogram of user-observed foreground latencies in our multi- fsync experiments. Each experiment is run for two minutes on an HDD. We bucketize the latencies in 200ms buckets in panel (a) and 100ms buckets in panels (b) and (c).

These results show that ccfs is effective at avoiding write dependence overheads when multiple streams are used (in comparison to a file system providing global order). The results also show that, within a stream, write dependence can cause noticeable overhead. For certain applications, therefore, it is possible that dividing the application into multiple streams is necessary for performance. The subsequent sections show that the majority of the applications do not require such division.

\subsection{Single-Stream Overheads}

The previous experiments show how ccfs avoids the performance overheads across streams; we now focus on performance within a stream. The performance effects of maintaining order within a stream are affected by false dependencies between updates within the stream, and hence depend significantly on the pattern of writes. We perform our evaluation using the Filebench [13, 55] suite that reflects real-world workload patterns and microbenchmarks, and compare performance between ext4 (false dependencies are not exposed) and ccfs (false dependencies are exposed because of ordering within streams). Another source of overhead within streams is the disk-level mechanism used to maintain order, that is, the SDJ technique used in ccfs. Hence, we compare performance between ext4 (no order), ccfs (order-preserving delayed allocation and SDJ), and ext4 in the data=journal mode (ext4- $d j$, full data journaling). We compare performance both with an HDD (disk-level overheads dominated by seeks) and an SSD (seeks less pronounced).

The overall results are shown in Figure 8; performance is most impacted by overwrites and fsync calls. We now explain the results obtained on each benchmark.

The varmail benchmark emulates a multithreaded mail server, performing file creates, appends, deletes, reads, and fsync calls in a single directory. Since each append is immediately followed by an fsync, there is no additional write dependence due to ordering. Performance is dominated by seek latency induced by the frequent fsync calls, resulting in similar performance across ext 4 and ccfs. Ext4-dj issues more writes but incurs fewer seeks (since data is written to the journal rather 


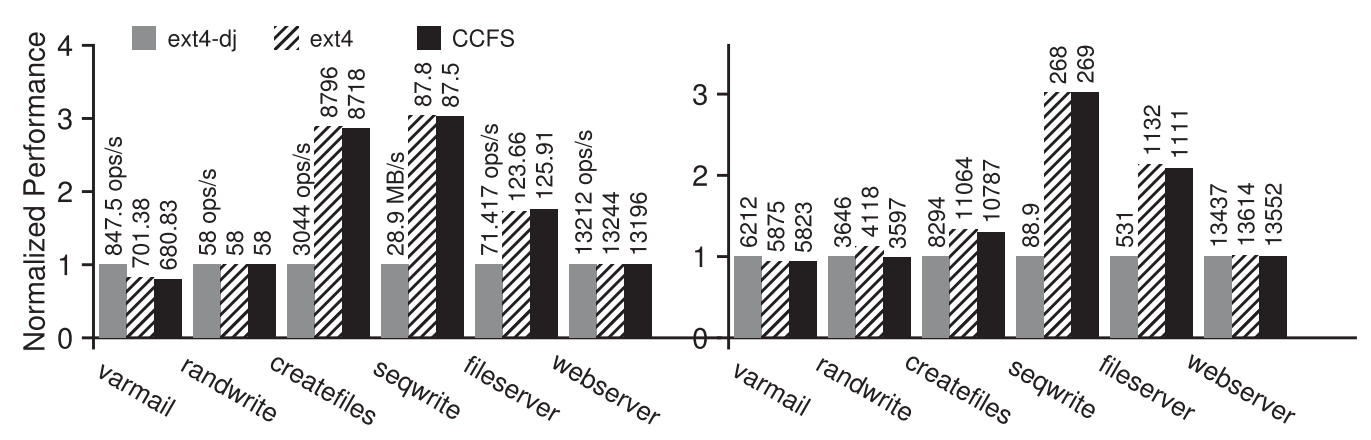

(a) HDD Performance

(b) SSD Performance

\begin{tabular}{c|r|r|r|r|r} 
& \multicolumn{3}{|c|}{ Writes } & \multicolumn{2}{c}{$\begin{array}{c}\text { CPU } \\
(K B / o p)\end{array}$} \\
\cline { 2 - 6 } & ext4-dj & ext4 & \multicolumn{1}{c|}{ ccfs } & ext4 & \multicolumn{1}{c}{ ccfs } \\
\hline varmail & 3.42 & 2.91 & 2.98 & 59.9 & 67.2 \\
randwrite & 8.10 & 4.10 & 8.10 & 16.8 & 24.4 \\
createfiles & 12.22 & 5.53 & 5.49 & 89.1 & 94.4 \\
seqwrite & 2.00 & 1.00 & 1.00 & 0.9 & 2.4 \\
fileserver & 1093.00 & 321.00 & 327.00 & 1040.0 & 2937.0 \\
webserver & 0.49 & 0.24 & 0.15 & 74.4 & 75.5
\end{tabular}

(c) HDD Writes and CPU usage

Fig. 8. Imposing Order at Disk-Level: Performance, Data Written, and CPU usage. Panels (a) and (b) show throughput under standard benchmarks for ccfs, ext4, and ext4 under the data=journal mode (ext4-dj), all normalized to ext4-dj. Panel (c) shows the total writes and CPU usage with an HDD. Varmail emulates a multithreaded mail server, performing file creates, appends, deletes, reads, and fsync in a single directory. Randwrite does 200,000 random writes (each of $4 K B$ in size) over a 10GB file with an fsync every 100 writes. Webserver emulates a multithreaded web server performing open-read-close on multiple files and a log file append. Createfiles uses 64 threads to create $1 M$ files, each of size $16 \mathrm{~KB}$. Seqwrite writes $32 G B$ to a new file (1KB is considered an operation in panel (c)). Fileserver emulates a file server, using 50 threads to perform creates, deletes, appends, and reads, on $80 \mathrm{~K}$ files. The fileserver, varmail, and webserver workloads were run for 300 s. The numbers reported are the average over 10 runs.

than the in-place location during each fsync), and performs $20 \%$ better in the HDD and 5\% better in the SSD.

Rand write overwrites random locations in an existing file and calls f sync every 100 writes. Since the fsync calls always flush the entire file, there is no additional write dependence due to ordering. However, the overwrites cause both ccfs (SDJ) and ext4-dj (full journaling) to write twice as much data as ext4. On the HDD, all file systems perform similarly, since seeks dominate performance; in the SSD, additional writes cause a $12 \%$ performance decrease for ccfs and ext4-dj.

Createfiles and seqwrite keep appending to files, while fileserver issues appends and deletes to multiple files; they do not perform any overwrites or issue any fsync calls. Since only appends are involved, ccfs writes the same amount of data as ext4. On the HDD, similar performance is observed in ccfs and in ext4. Under SSDs, createfiles is $4 \%$ slower atop ccfs because of delayed allocation in the T_LOCKED state, which takes a noticeable amount of time (an average of $132 \mathrm{~ms}$ during each commit); this is an implementation artifact and can be optimized. For all these benchmarks, ext4-dj writes data twice and hence is significantly slower. Webserver involves mostly reads and a few appends; performance is dominated by reads, so all file systems perform similarly. 
Figure 8(c) compares the CPU usage of ccfs and ext4. For most workloads, our current implementation of ccfs has moderately higher CPU usage; the significant usage for fileserver and seqwrite is because the workloads are dominated by block allocations and de-allocations, which are especially CPU-intensive for our implementation. This can be improved by adopting more optimized structures and lookup tables (Section 3.5). Thus, while it does not noticeably impact performance in our experiments, reducing CPU usage is an important future goal for ccfs.

Overall, our results show that maintaining order does not incur any inherent performance overhead for standard workloads when the workload is run in one stream. False dependencies are rare and have little impact for common workloads, and the technique used to maintain order within streams in ccfs is efficient.

\subsection{Case Studies}

Our evaluation in the previous section shows the performance effects of maintaining order for standard benchmarks. We now consider three real-world applications: Git, LevelDB, and SQLite with rollback journaling; we focus on the effort required to maintain crash consistency with good performance for these applications in ccfs and the default mode (data=ordered) of ext4. For ext4, we ensure that the applications remain consistent by either modifying the application to introduce additional fsync calls or using safe application configuration options. All three applications are naturally consistent on ccfs when run on a single stream.

Single-Application Performance. We first ran each application in its own stream in the absence of other applications, to examine if running the application in one stream is sufficient for good performance (as opposed to dividing a single application into multiple streams). Specifically, we try to understand if the applications have false dependencies. We also consider their performance when fsync calls are omitted without affecting consistency (including user-visible durability) on ccfs.

The results are shown in Table 5. For Git, we use a workload that adds and commits the Linux source code to an empty repository. While Git is naturally consistent atop ccfs, it requires a special option ( $f$ syncobjectfiles) on ext4; this option causes Git to issue many fsync calls. Irrespective of this option, Git always issues $242 \mathrm{MB}$ of appends and no overwrites. In ccfs, the $242 \mathrm{MB}$ is sent directly to the device and the workload completes in 28.9 s. In ext4, the fsync calls needed for correctness prevent updates to metadata blocks from being coalesced; for example, a block bitmap that is repeatedly updated by the workload needs to be written to the journal on every fsync. Moreover, each fsync call forces a separate journal transaction, writing a separate descriptor block and commit block to the disk and causing two disk cache flushes. Thus, in ext4, the workload results in $1.4 \mathrm{~GB}$ of journal commits and takes $2,294 \mathrm{~s}$ to complete ( $80 \times$ slower).

For SQLite, we insert 2,000 rows of 120 bytes each into an empty table. SQLite issues fsync calls frequently, and there are no false dependencies in ccfs. However, SQLite issues file overwrites (31.83MB in this workload), which causes data to be sent to the journal in ccfs. Sending the overwritten data to the journal improves the performance of ccfs in comparison to ext4 (1.28 $\times)$. Because SQLite frequently issues an fsync after overwriting a small amount (4KB) of data, ext4 incurs a seek during each fsync call, which ccfs avoids by writing the data to the journal. SQLite can also be heavily optimized when running atop ccfs by omitting unnecessary fsync calls; with our workload, this results in a $685 \times$ improvement.

For LevelDB, we use the fillrandom benchmark from the db_bench tool to insert 250K key-value pairs of 1,000 bytes each to an empty database. Atop ext4, we needed to add additional fsync calls to improve the crash consistency of LevelDB. LevelDB on ccfs and the fixed version on ext4 have similar write avoidance, as can be seen from Table 5. Since LevelDB also does few file overwrites, 
Table 5. Case Study: Single-Application Performance

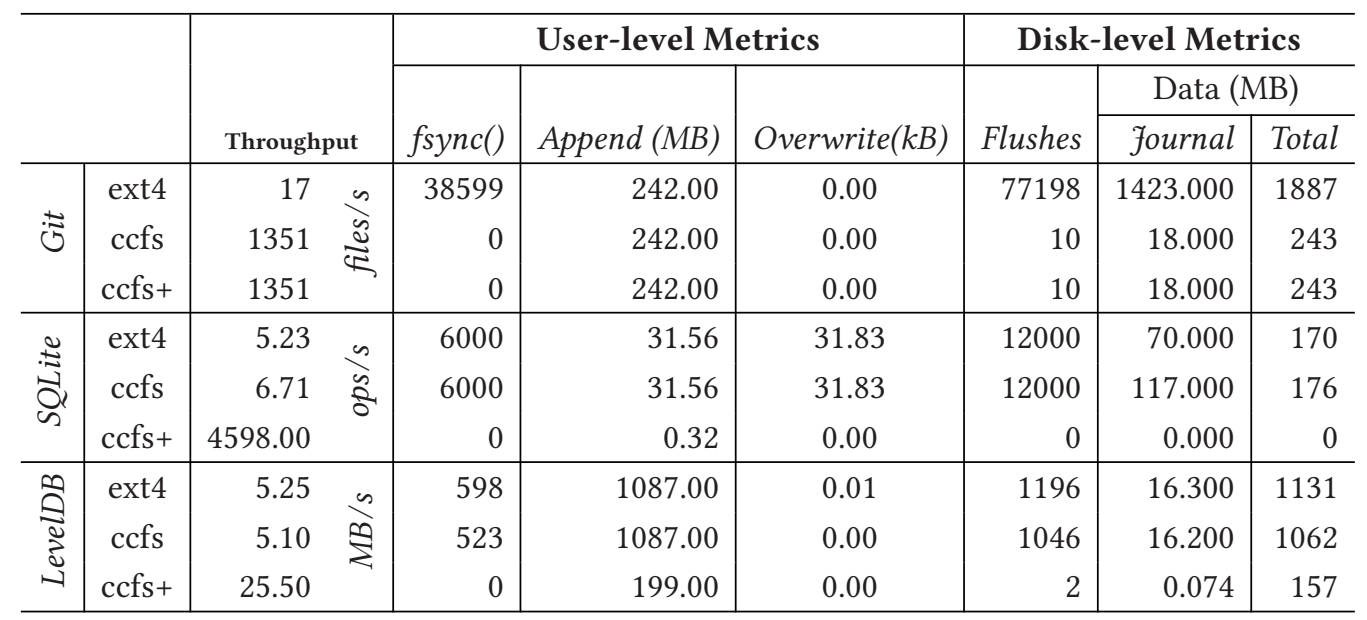

The table shows the performance and observed metrics of Git, LevelDB, and SQLite-rollback run separately under different filesystem configurations on HDD. ccfs+ denotes running ccfs with unnecessary fsynccalls omitted; in both ccfs configurations, the application tuns in a single stream. The user-level metrics characterize each workload; "appends" and "overwrites" show how much appended and overwritten data needs to be flushed by fsynccalls (and also how much remain buffered when the workload ends). Overhead imposed by maintaining order will be observed by fsynccalls in the ccfs configuration needing to flush more data. The disk-level metrics relate the characteristics to actual data written to the device.

it performs similarly on ccfs and ext4. With ccfs, existing fsync calls in LevelDB can be omitted, since ccfs already provides ordering, increasing performance $5 \times$.

Thus, the experiments suggest that false-dependency overheads are minimal within an application. In two of the applications, the ordering provided by ccfs can be used to omit fsync calls to improve performance.

Multiple-Application Performance. We next test whether ccfs is effective in separating streams: Figure 9 shows the performance when running Git and SQLite simultaneously. The situation in current real-world deployments is exemplified by the ext4-bad configuration in Figure 9: both applications are run on ext4, but Git runs without the fsyncobjectfiles option (i.e., consistency is sacrificed). The $c c f s-2$ configuration is the intended use case for ccfs: Git and SQLite are in separate streams on ccfs, achieving consistency while performing similarly to ext4bad. (SQLite performs better under $c c f s-2$, because ccfs sends some data to the journal and reduces seeks, as explained previously.) Thus, ccfs achieves real-world performance while improving correctness.

The $c c f s-1$ configuration demonstrates the overhead of global order by running Git and SQLite in the same stream on ccfs; this is not the intended use case of ccfs. This configuration heavily impacts SQLite's performance because of (false) dependencies introduced from writes issued by Git. Running applications in separate streams can thus be necessary for acceptable performance.

The ext 4 configuration reiterates previous findings: it maintains correctness using Git's $f$ syncobjectfiles on ext 4 , but Git is unacceptably slow due to fsync calls. The $c c f s+$ configuration represents a secondary use case for ccfs: it runs the applications in separate streams on ccfs with unneeded fsync calls omitted, resulting in better SQLite performance (Git is moderately slower, since SQLite uses more disk bandwidth). Thus, running each application in its stream achieves correctness with good performance, while global order achieves correctness but reduces performance. 


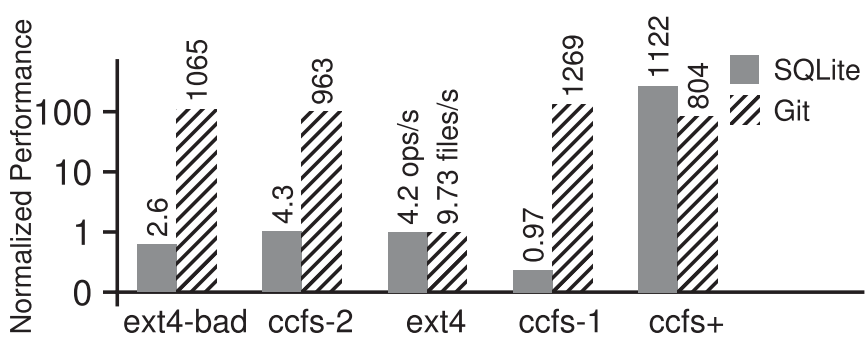

Fig. 9. Case Study: Multiple-Application Performance. The figure shows the performance of Git and SQLiterollback run simultaneously under different configurations on HDD, normalized to performance under the ext4 configuration (higher is better). The ext4-bad configuration runs the applications on ext4, with consistency sacrificed in Git. ccfs-2 uses separate streams for each application on ccfs. Ext4 uses ext4 with consistent Git. ccfs-1 runs both applications in the same stream on ccfs. ccfs+ runs applications in separate streams without unnecessary fsync calls. Workload: Git adds and commits a repository 25 times the size of Linux; SQLite repeatedly inserts 120-byte rows until Git completes.

Overall, all our performance experiments show that ccfs provides in-order update semantics while achieving performance similar to a re-ordering file system, confirming our efficiency hypothesis.

\subsection{Developer Effort}

We now discuss the developer effort required to make an application crash consistent atop ccfs and other re-ordering file systems. Figure $10(\mathrm{a}(\mathrm{i})$ ) shows the code changes needed to make Git crash consistent atop ccfs. Achieving correctness atop ccfs (while maintaining performance) required negligible developer overhead: we added a single line to the main function that sets the stream using the setstream() call. Similarly, for all other applications, we added one setstream() call to the beginning of each application, without examining the applications any further. To omit unnecessary fsync calls in ccfs and improve performance (i.e., for the ccfs + configuration), we used the IGNORE_FSYNC flag on the setstream() calls, and added streamsync() calls where durability is desired. Identifying such locations for streamsync() calls was straightforward: we added them to places in the code where the user is guaranteed durability (i.e., transaction commit points). Specifically, we added the streamsync() calls in one and two places in LevelDB and SQLite, respectively.

Note that the change shown in Figure 10(a(i)) makes all Git processes share the same stream, since the same string ("git") is used to initialize the stream. Assigning Git processes that work on different repositories to different streams is also straightforward: one can simply use the repository path as the stream identifier, as shown in Figure 10(a(ii)). We note here that we do not discuss in detail the security issues associated with using simple stream identifiers. For example, an application could affect another application's performance by deliberately using the same identifier. However, one could tackle this problem by using a file-system pathname as the identifier and its associated permissions to prevent other applications from using the same identifier.

Achieving crash consistency atop re-ordering file systems required considerably more developer effort. For example, correctness with ext4 required two additional fsync calls on LevelDB and the fsyncobjectfiles option on Git. The changes in ext 4 both reduced performance and were complicated; we carefully used results from the Alice study to determine the additional fsync calls necessary for correctness.

Note that, while we happened to find that Git's fsyncobjectfiles makes it correct on ext4, other changes are needed for other file systems such as btrfs and ext2. Figure 10(b) shows the code 


\section{DISCUSSION}

The stream abstraction and ccfs have been designed to significantly improve crash consistency and performance, with minimal developer effort, in a broad range of applications. Consequently, there are specific applications for which the abstraction and the current implementation of ccfs are not ideal.

In this section, we discuss three separate aspects that determine whether a given application would find streams useful in practice. We first outline how the stream abstraction can be a fundamental mismatch for certain applications because of false dependencies. We then discuss how the abstraction imposes a trade-off on all file systems that implement streams, and how this affects applications. We finally explain specific limitations of ccfs that can possibly degrade application performance.

\subsection{False Dependencies}

False dependencies are a fundamental aspect in the stream abstraction that can cause performance overhead. In theory, for any application, one can always construct streams with no false dependencies by determining sets of writes that really require sequential order among them. In the extreme, each write can be associated with its own stream (note that fsync-like calls already in the application are still enforced, and consistency is not affected).

In practice, in many application workloads, most false dependencies can be avoided with little developer effort by associating entire threads to each stream (as shown in the previous section). But this strategy does not work in some applications, especially in applications where multiple layers of code hide the logical relationships between writes. As an example, consider a type-2 hypervisor that stripes multiple guest-level drives across many host-level files written by a single thread. It might be impractical for the hypervisor thread to use streams while avoiding false dependencies (note that streams can still be used within the guest OS): the natural relationships between individual writes inside the guest operating systems are forgotten by the time those writes reach host-level files. Streams are not ideal in such applications because of the developer effort involved in separating out related sets of writes into different streams.

\subsection{File System Trade-Offs}

Even in the absence of false dependencies, the stream abstraction imposes overhead due to the guarantee of sequential ordering within a stream. For example, consider the following code sequence: write $\left(f_{1}\right.$, "hello"); write $\left(f_{1}\right.$, "world"); fsync $\left(f_{1}\right)$;. Write avoidance is not affected by streams, since in any file system, we expect hello and world to be persistent on the disk when the fsync returns. There are hence no false dependencies according to our previous definition (Section 2.3). But, with streams, we expect hello to be persistent before world, an additional requirement over ordinary file systems that can cost performance.

File systems that implement streams, therefore, have to use techniques to hide the performance overhead of maintaining order. We know of two basic techniques that allow the appearance of sequential order to be enforced efficiently (without explicit hardware support): journaling and copyon-write (or log structuring). While both approaches still pose overheads (writing twice and affecting data locality, respectively), file systems can adopt different optimizations and make trade-offs for maintaining order. For example, ccfs avoids any overhead for appended data and chooses journaling over copy-on-write for overwritten data; a more complex file system could introduce additional optimizations, such as learning from workload characteristics and then dynamically choosing between journaling and copy-on-write for overwritten data. Thus, the ordering technique 
should not affect application performance in practice; we believe file systems can be constructed to make the overhead insignificant for most workloads, as evidenced in the previous section.

However, there remains a practical concern for some application workloads even with efficient file-system design: the need to adopt significant techniques for maintaining sequential order, along with trade-offs and optimizations, changes disk-level behavior from the perspective of an application. For example, assuming the read and write performance behavior of a hard-drive, a database might use an optimized B-tree structure for efficiency; a file system implementing streams will change that behavior with its ordering technique. Furthermore, different file systems can make different trade-offs to maintain order, and can adopt different optimizations. Streams might hence not be ideal for applications that assume particular disk-level effects from writes, such as heavily optimized traditional databases.

\subsection{CCFS-Specific Effects}

The current implementation of ccfs has limitations. First, it journals overwritten data, and applications that heavily overwrite data might find performance degraded. As previously explained, this could be improved by more optimizations, such as using copy-on-write when multiple contiguous blocks are overwritten (journaling can actually improve performance with random writes [41]).

Second, ccfs uses linear directories in the pursuit of pointer-less data structures (ext 4 uses a hash-tree structure as its default). Although we did not observe any performance effects due to this in our evaluation, directory-heavy workloads could be affected. If necessary, however, then ccfs directories could be further optimized; for example, the linear directory currently implemented could be periodically compacted into a hash-tree in the background, in a manner reminiscent of LSM-trees.

\subsection{Summary}

While streams are useful for most applications, it is important to recognize that they are not compatible with all applications. Application incompatibility can arise from the stream abstraction itself, or due to the implementation of a particular file system. We identified two example applications in this section that are not ideal considering the abstraction itself: type- 2 hypervisors (because of false dependencies) and traditional relational databases (because streams make disklevel effects less transparent). Note that both are significantly complex applications that might detect and be optimized for very specific device characteristics; it is reasonable to expect that they spent significant effort to verify their crash consistency. We believe that, unlike such complex applications, for applications that actually desire streams to improve consistency or performance, the abstraction will not impose overhead.

We also identified in this section that the ordering technique is an important design choice for file systems that implement streams, and explained how a combination of journaling and copyon-write is a good choice. We considered two specific limitations of ccfs (journaling overwritten data and linear directories) in this section, and suggested alternatives for them. In general, while file systems that implement streams need to be more complex, sufficient optimizations can be introduced to make any overhead insignificant.

\section{RELATED WORK}

We briefly describe how ccfs differs from previous work:

Atomicity interfaces. Transactional file-system interfaces have a long history [48] and allow applications to delegate most crash-consistency requirements to the file system. Recent work in this space includes file systems providing ACID semantics such as Amino [59], Valor [52], and 
Windows TxF [30], atomicity-only file systems as proposed by Vermat et al. [58], Park et al. [35], and CFS [31], and OS-level transaction support as advocated by TxOS [40]. Such interfaces allow adding crash consistency easily to applications that do not already implement them, and help heavily optimized applications that trade portability for performance [28].

For applications with existing consistency implementations, proponents of atomicity interfaces and transactional file systems advocate replacing the existing implementation with the interface provided by the file system. This is not trivial to achieve (though perhaps much easier than writing a new consistency implementation). For instance, consider the SQLite database, and assume that we replace its consistency implementation using a straightforward begin_atomic()-end_atomic() interface provided by the file system. This does not work for two reasons. First, it does not offer SQLite's ROLLBACK command [54] (i.e., abort transaction) and the SAVEPOINT command (which allows an aborted transaction to continue from a previous point in the transaction). Second, unless the file system provides isolation (which recent research argues against [31]), it requires reimplementing isolation and concurrency control, since SQLite's isolation mechanism is inherently tied to its consistency mechanism [53]. With applications such as LevelDB, where the consistency mechanism is tightly coupled to query-efficient on-disk data structures [26, 34], adopting alternative consistency mechanisms will also cause unnecessary performance changes.

To summarize, adopting atomicity interfaces to overcome vulnerabilities is nonoptimal in applications with existing consistency implementations. One challenge is simply the changes required: CFS [31], with arguably the most user-friendly atomic interface, requires changing 38 lines in SQLite and 240 lines in MariaDB. Another challenge is portability: until the interfaces are widely available, the developer must maintain both the existing consistency protocol and a protocol using the atomic interface; this has deterred such interfaces in Linux [10]. Finally, the complexity of data structures and concurrency mechanisms in modern applications (e.g., LSM trees) are not directly compatible with a generic transactional interface; Windows TxF, a transactional interface to NTFS, is being considered for deprecation due to this [30]. In contrast, streams focus on masking vulnerabilities in existing application-level consistency implementations. Ccfs advocates a single change to the beginning of applications and running them without more modification on both stream-enabled and stream-absent file systems.

Ordering interfaces. Fine-grained ordering interfaces $[5,6,14]$ supplement the existing fsync call, making it less costly for applications to easily achieve crash consistency. They allow better performance, but require developers to specify the exact ordering required, and as such are not optimal for fixing existing protocol implementations. Ext4's data-journaled mode and LinLogFS [11] provide a globally ordered interface, but incur unacceptable disk-level ordering and false-dependence overhead. Xsyncfs [33] provides global order and improves performance by buffering user-visible outputs; this approach is complementary to our approach of reducing false dependencies. Other proposed ordering interfaces $[9,36]$ focus only on NVMs.

Implementation. Ccfs builds upon seminal work in database systems [18, 32] and file-system crash consistency $[8,12,15,16,19,44,46,49]$, but is unique in assembling different techniques needed for efficient implement of the stream API. Specifically, ccfs uses journaling $[8,19]$ for order within a stream but applies techniques similar to soft updates [15, 16, 49] for separating streams. Such a design is necessary: using soft updates directly for a long chain of dependent writes ordered one after the other (as ccfs promises within a stream) will result in excessive disk seeks. Block-level guarantees of atomicity and isolation, such as Isotope [50] and TxFlash [42], can simplify separation of streams in ccfs; however, techniques in Section 3 are still necessary. IceFS [27] extends ext3 to support multiple virtual journals, but requires data journaling within each journal to support ordered data writes, and hence cannot be directly used to improve application consistency without 
reducing performance. IceFS also does not use techniques similar to soft updates to separate the virtual journals, associating only a static and coarse-grained partition of the file-system namespace to each journal (compared to the dynamic and fine-grained stream abstraction).

In principle, one should be able to easily construct a stream-ordered file system atop a finegrained ordering interface. However, the direct implementation of ordering in Featherstitch [14] uses the soft-updates approach, which is incompatible as described. The interface provided by OptFS [6] is insufficient for implementing streams. Ccfs uses the selective data journaling (SDJ) technique from OptFS but optimizes it; the original relies on specialized hardware (durability notifications) and decreased guarantees (no durability) for efficiency.

\section{CONCLUSION}

In this article, we present the stream abstraction as a practical solution for application-level crash consistency. We describe the stream API and the ccfs file system, an efficient implementation of the API. We confirm the ordering hypothesis by showing that crash consistency in real applications can be significantly improved by ccfs's in-order update and weak atomicity semantics. We confirm our efficiency hypothesis by comparing ccfs's performance with ext4, finding that ccfs maintains (and sometimes significantly improves) performance while improving correctness. Our results suggest that developer effort for using the streams API is negligible and practical.

\section{ACKNOWLEDGMENTS}

We thank the anonymous reviewers and Ashvin Goel (our shepherd for the FAST version of the article) for their insightful comments and suggestions. We thank the members of the Advanced Systems Laboratory (ADSL) for their valuable discussions.

\section{REFERENCES}

[1] Ramnatthan Alagappan, Aishwarya Ganesan, Yuvraj Patel, Thanumalayan Sankaranarayana Pillai, Andrea C. ArpaciDusseau, and Remzi H. Arpaci-Dusseau. 2016. Correlated crash vulnerabilities. In Proceedings of the 12th Symposium on Operating Systems Design and Implementation (OSDI'16).

[2] Remzi H. Arpaci-Dusseau and Andrea C. Arpaci-Dusseau. 2014. Operating Systems: Three Easy Pieces (0.9 ed.). ArpaciDusseau Books.

[3] Andrew D. Birrell. 1989. An Introduction to Programming with Threads. Technical Report SRC-RR-35.

[4] James Bornholt, Antoine Kaufmann, Jialin Li, Arvind Krishnamurthy, Emina Torlak, and Xi Wang. 2016. Specifying and checking file system crash-consistency models. In Proceedings of the 21st International Conference on Architectural Support for Programming Languages and Operating Systems (ASPLOS'16).

[5] Nathan C. Burnett. 2006. Information and Control in File System Buffer Management. Ph.D. Dissertation. University of Wisconsin-Madison.

[6] Vijay Chidambaram, Thanumalayan Sankaranarayana Pillai, Andrea C. Arpaci-Dusseau, and Remzi H. ArpaciDusseau. 2013. Optimistic crash consistency. In Proceedings of the 24th ACM Symposium on Operating Systems Principles (SOSP'13).

[7] Vijay Chidambaram, Tushar Sharma, Andrea C. Arpaci-Dusseau, and Remzi H. Arpaci-Dusseau. 2012. Consistency without ordering. In Proceedings of the 10th USENIX Symposium on File and Storage Technologies (FAST'12). 101-116.

[8] Sailesh Chutani, Owen T. Anderson, Michael L. Kazar, Bruce W. Leverett, W. Anthony Mason, and Robert N. Sidebotham. 1992. The episode file system. In Proceedings of the USENIX Winter Technical Conference (USENIX Winter'92). 43-60.

[9] Jeremy Condit, Edmund B. Nightingale, Christopher Frost, Engin Ipek, Benjamin Lee, Doug Burger, and Derrick Coetzee. 2009. Better I/O through byte-addressable, persistent memory. In Proceedings of the 22nd ACM Symposium on Operating Systems Principles (SOSP'09).

[10] Jonathan Corbet. 2009. Better than POSIX? March 2009. Retrieved April 2016 from https://lwn.net/Articles/323752/.

[11] Christian Czezatke and M. Anton Ertl. 2000. LinLogFS: A log-structured filesystem for linux. In Proceedings of the USENIX Annual Technical Conference (FREENIX Track'00).

[12] Linux Documentation. 2016. XFS Delayed Logging Design. Retrieved April 2016 from https://www.kernel.org/doc/ Documentation/filesystems/xfs-delayed-logging-design.txt. 
[13] Filebench. 2016. Filebench. Retrieved March 2016 from https://github.com/filebench/filebench/wiki.

[14] Christopher Frost, Mike Mammarella, Eddie Kohler, Andrew de los Reyes, Shant Hovsepian, Andrew Matsuoka, and Lei Zhang. 2007. Generalized file system dependencies. In Proceedings of the 21st ACM Symposium on Operating Systems Principles (SOSP’07). 307-320.

[15] Gregory R. Ganger, Marshall Kirk McKusick, Craig A. N. Soules, and Yale N. Patt. 2000. Soft updates: A solution to the metadata update problem in file systems. ACM Trans. Comput. Syst. (TOCS) 18, 2 (May 2000), 127-153.

[16] Gregory R. Ganger and Yale N. Patt. 1994. Metadata update performance in file systems. In Proceedings of the 1st Symposium on Operating Systems Design and Implementation (OSDI'94). 49-60.

[17] Kourosh Gharachorloo, Daniel Lenoski, James Laudon, Phillip Gibbons, Anoop Gupta, and John Hennessy. 1992. Memory consistency and event ordering in scalable shared-memory multiprocessors. In Proceedings of the 17th Annual International Symposium on Computer Architecture (ISCA'90). Seattle, WA.

[18] Jim Gray and Andreas Reuter. 1993. Transaction Processing: Concepts and Techniques. Morgan Kaufmann.

[19] Robert Hagmann. 1987. Reimplementing the cedar file system using logging and group commit. In Proceedings of the 11th ACM Symposium on Operating Systems Principles (SOSP'87).

[20] Timothy L. Harris. 2001. A Pragmatic Implementation of Non-blocking Linked-lists. DISC.

[21] Tyler Harter, Chris Dragga, Michael Vaughn, Andrea C. Arpaci-Dusseau, and Remzi H. Arpaci-Dusseau. 2011. A file is not a file: Understanding the I/O behavior of apple desktop applications. In Proceedings of the 23rd ACM Symposium on Operating Systems Principles (SOSP'11).

[22] Maurice Herlihy. 1991. Wait-free synchronization. Transactions on Programming Languages 11, 1 (January 1991).

[23] D. M. Jacobson and J. Wilkes. 1991. Disk Scheduling Algorithms Based on Rotational Position. Technical Report HPLCSP-91-7. Hewlett Packard Laboratories.

[24] Jaeho Kim, Jongmoo Choi, Yongseok Oh, Donghee Lee, Eunsam Kim, and Sam H. Noh. 2009. Disk schedulers for solid state drives. In EMSOFT. Grenoble, France.

[25] Lanyue Lu, Andrea C. Arpaci-Dusseau, Remzi H. Arpaci-Dusseau, and Shan Lu. 2013. A study of linux file system evolution. In Proceedings of the 11th USENIX Symposium on File and Storage Technologies (FAST'13). San Jose, CA

[26] Lanyue Lu, Thanumalayan Sankaranarayana Pillai, Andrea C. Arpaci-Dusseau, and Remzi H. Arpaci-Dusseau. 2016. WiscKey: Separating keys from values in SSD-conscious storage. In Proceedings of the 14th USENIX Conference on File and Storage Technologies (FAST'16).

[27] Lanyue Lu, Yupu Zhang, Thanh Do, Samer Al-Kiswany, Andrea C. Arpaci-Dusseau, and Remzi H. Arpaci-Dusseau. 2014. Physical disentanglement in a container-based file system. In Proceedings of the 11th Symposium on Operating Systems Design and Implementation (OSDI'14).

[28] MariaDB. 2016. Fusion-io NVMFS Atomic Write Support. Retrieved April 2016 from https://mariadb.com/kb/en/ mariadb/fusion-io-nvmfs-atomic-write-support/.

[29] Mercurial. 2016. Dealing with Repository and Dirstate Corruption. Retrieved April 2016 from https://www. mercurial-scm.org/wiki/RepositoryCorruption.

[30] Microsoft. 2016. Alternatives to using Transactional NTFS. Retrieved April 2016 from https://msdn.microsoft.com/ en-us/library/hh802690.aspx.

[31] Changwoo Min, Woon-Hak Kang, Taesoo Kim, Sang-Won Lee, and Young Ik Eom. 2015. Lightweight application-level crash consistency on transactional flash storage. In Proceedings of the USENIX Annual Technical Conference (USENIX ATC'15).

[32] C. Mohan, D. Haderle, B. Lindsay, H. Pirahesh, and P. Schwarz. 1992. ARIES: A transaction recovery method supporting fine-granularity locking and partial rollbacks using write-ahead logging. ACM Trans. Data. Syst. 17, 1 (March 1992), 94-162.

[33] Edmund B. Nightingale, Kaushik Veeraraghavan, Peter M. Chen, and Jason Flinn. 2006. Rethink the sync. In Proceedings of the 7th Symposium on Operating Systems Design and Implementation (OSDI'06).

[34] Patrick O'Neil, Edward Cheng, Dieter Gawlick, and Elizabeth O’Neil. 1996. The log-structured merge-tree (LSM-tree). Acta Informat. 33, 4 (1996), 351-385.

[35] Stan Park, Terence Kelly, and Kai Shen. 2013. Failure-atomic msync (): A simple and efficient mechanism for preserving the integrity of durable data. In Proceedings of the EuroSys Conference (EuroSys'13).

[36] Steven Pelley, Peter M. Chen, and Thomas F. Wenisch. 2014. Memory persistency. In Proceedings of the 41st International Symposium on Computer Architecture (ISCA'14).

[37] Thanumalayan Sankaranarayana Pillai, Ramnatthan Alagappan, Lanyue Lu, Vijay Chidambaram, Andrea C. ArpaciDusseau, and Remzi H. Arpaci-Dusseau. 2017. Application crash consistency and performance with CCFS. In Proceedings of the 15th USENIX Conference on File and Storage Technologies (FAST'17).

[38] Thanumalayan Sankaranarayana Pillai, Vijay Chidambaram, Ramnatthan Alagappan, Samer Al-Kiswany, Andrea C. Arpaci-Dusseau, and Remzi H. Arpaci-Dusseau. 2014. All file systems are not created equal: On the complexity 
of crafting crash-consistent applications. In Proceedings of the 11th Symposium on Operating Systems Design and Implementation (OSDI'14).

[39] Thanumalayan Sankaranarayana Pillai, Vijay Chidambaram, Ramnatthan Alagappan, Samer Al-Kiswany, Andrea C. Arpaci-Dusseau, and Remzi H. Arpaci-Dusseau. 2015. Crash consistency. Commun. ACM 58, 10 (October 2015).

[40] Donald E. Porter, Owen S. Hofmann, Christopher J. Rossbach, Alexander Benn, and Emmett Witchel. 2008. Operating systems transactions. In Proceedings of the 8th Symposium on Operating Systems Design and Implementation (OSDI'08).

[41] Vijayan Prabhakaran, Andrea C. Arpaci-Dusseau, and Remzi H. Arpaci-Dusseau. 2005. Analysis and evolution of journaling file systems. In Proceedings of the USENIX Annual Technical Conference (USENIX'05). 105-120.

[42] Vijayan Prabhakaran, Thomas L. Rodeheffer, and Lidong Zhou. 2008. Transactional flash. In Proceedings of the 8th Symposium on Operating Systems Design and Implementation (OSDI'08).

[43] Benjamin Recht, Christopher Re, Stephen Wright, and Feng Niu. 2011. Hogwild: A Lock-free Approach to Parallelizing Stochastic Gradient Descent. Advances in Neural Information Processing (2011), 693-701.

[44] Mendel Rosenblum and John Ousterhout. 1992. The design and implementation of a log-structured file system. ACM Trans. Comput. Syst. 10, 1 (February 1992), 26-52.

[45] Chris Ruemmler and John Wilkes. 1994. An introduction to disk drive modeling. IEEE Comput. 27, 3 (March 1994), 17-28.

[46] Margo Seltzer, Keith Bostic, Marshall Kirk McKusick, and Carl Staelin. 1993. An implementation of a log-structured file system for UNIX. In Proceedings of the USENIX Winter Technical Conference (USENIX Winter'93). 307-326.

[47] Margo Seltzer, Peter Chen, and John Ousterhout. 1990. Disk scheduling revisited. In Proceedings of the USENIX Winter Technical Conference (USENIX Winter'90). 313-324.

[48] Margo I. Seltzer. 1993. File System Performance and Transaction Support. Ph.D. Dissertation. EECS Department, University of California, Berkeley.

[49] Margo I. Seltzer, Gregory R. Ganger, M. Kirk McKusick, Keith A. Smith, Craig A. N. Soules, and Christopher A. Stein. 2000. Journaling versus soft updates: Asynchronous meta-data protection in file systems. In Proceedings of the USENIX Annual Technical Conference (USENIX'00). 71-84.

[50] Ji-Yong Shin, Mahesh Balakrishnan, Tudor Marian, and Hakim Weatherspoon. 2016. Isotope: Transactional isolation for block storage. In Proceedings of the 14th USENIX Conference on File and Storage Technologies (FAST'16).

[51] Daniel J. Sorin, Mark D. Hill, and David A. Wood. 2011. A Primer on Memory Consistency and Cache Coherence. Synthesis Lectures on Computer Architecture, 1st Morgan \& Claypool (November 2011).

[52] Richard P. Spillane, Sachin Gaikwad, Manjunath Chinni, Erez Zadok, and Charles P. Wright. 2009. Enabling transactional file access via lightweight kernel extensions. In Proceedings of the 7th USENIX Symposium on File and Storage Technologies (FAST'09).

[53] SQLite. 2016. Isolation In SQLite. Retrieved Dec 2016 from https://www.sqlite.org/isolation.html.

[54] SQLite. 2016. SQL As Understood By SQLite. Retrieved Dec 2016 from https://www.sqlite.org/lang.html.

[55] Vasily Tarasov, Erez Zadok, and Spencer Shepler. 2016. Filebench: A flexible framework for file system benchmarking. ;login: USENIX Mag. 41, 1 (June 2016).

[56] Linus Torvalds. 2009. Linux 2.6.29. Retrieved April 2016 from https://lkml.org/lkml/2009/3/25/632.

[57] Theodore Ts'o. 2012. ext4: remove calls to ext4_jbd2_file_inode() from delalloc write path. Retrieved April 2016 from http://lists.openwall.net/linux-ext4/2012/11/16/9.

[58] Rajat Verma, Anton Ajay Mendez, Stan Park, Sandya Srivilliputtur Mannarswamy, Terence P. Kelly, and Charles B. Morrey III. 2015. Failure-atomic updates of application data in a linux file system. In Proceedings of the 13th USENIX Conference on File and Storage Technologies (FAST'15).

[59] Charles P. Wright, Richard Spillane, Gopalan Sivathanu, and Erez Zadok. 2007. Extending ACID semantics to the file system via ptrace. ACM Trans. Storage (TOS) 3, 2 (June 2007), 1-42.

[60] Junfeng Yang, Can Sar, and Dawson Engler. 2006. EXPLODE: A lightweight, general system for finding serious storage system errors. In Proceedings of the 7th Symposium on Operating Systems Design and Implementation (OSDI'06).

[61] Mai Zheng, Joseph Tucek, Dachuan Huang, Feng Qin, Mark Lillibridge, Elizabeth S. Yang, Bill W. Zhao, and Shashank Singh. 2014. Torturing databases for fun and profit. In Proceedings of the 11th Symposium on Operating Systems Design and Implementation (OSDI'14).

Received June 2017; revised June 2017; accepted June 2017 